\title{
A Constrained Many-objective Optimization Evolutionary Algorithm with Enhanced Mating and Environmental Selections
}

\author{
Fei Ming, Wenyin Gong, Ling Wang, and Liang Gao
}

\begin{abstract}
Unlike the considerable research on solving manyobjective optimization problems with evolutionary algorithms, there has been much less research on constrained many-objective optimization problems (CMaOPs). Generally, to effectively solve CMaOPs, an algorithm needs to balance feasibility, convergence, and diversity simultaneously. It is essential for handling CMaOPs yet most of the existing research encounters difficulties. This paper proposes a novel constrained many-objective optimization evolutionary algorithm with enhanced mating and environmental selections, namely CMME. The main features are: i) two ranking strategies are proposed and applied in the mating and environmental selections to enrich feasibility and convergence; ii) an individual density estimation is designed, and crowding distance is integrated to promote diversity; and iii) the $\theta$-dominance is used to strengthen the selection pressure on both the convergence and diversity. The synergy of these components can achieve the goal of balancing feasibility, convergence, and diversity for solving CMaOPs. The proposed CMME algorithm is evaluated on 10 CMaOPs with different features and a variable number of objective functions. Experimental results on three benchmark CMOPs and three real-world applications demonstrate that CMME shows superiority or competitiveness over nine related algorithms.
\end{abstract}

Index Terms-Constrained many-objective optimization, evolutionary algorithm, mating selection, environmental selection, ranking.

\section{INTRODUCTION}

C ONSTRAINED multi-objective optimization problems (CMOPs) appear in many real-world scenarios, such as vehicle routing problems [1], optimal scheduling of energy storage systems [2], and web service location allocation problems [3]. Generally, a CMOP can be formulated as:

$$
\begin{aligned}
\operatorname{minimize} & \mathbf{f}(\mathbf{x})=\left(f_{1}(\mathbf{x}), \cdots, f_{m}(\mathbf{x})\right)^{T} \\
\text { subject to } & \mathbf{x} \in \mathbb{S} \\
& g_{j}(\mathbf{x}) \leq 0, j=1, \cdots, p \\
& h_{j}(\mathbf{x})=0, j=p+1, \cdots, q
\end{aligned}
$$

This work was partly supported by the National Natural Science Foundation of China under Grant Nos. 62076225 and 62073300, the Natural Science Foundation for Distinguished Young Scholars of Hubei under Grant No. 2019CFA081, and the Fundamental Research Funds for the Central Universities, China University of Geosciences (Wuhan) under Grant no. CUGGC03. (Corresponding authors: Wenyin Gong \& Ling Wang.)

F. Ming and W. Gong are with School of Computer Science, China University of Geosciences, Wuhan 430074, China. (Email: wygong@ @ cug.edu.cn)

L. Wang is with Department of Automation, Tsinghua University, Beijing 100084, China. (Email: wangling@ @tsinghua.edu.cn)

L. Gao is with State Key Lab of Digital Manufacturing Equipment \& Technology, Huazhong University of Science and Technology, Wuhan, 430074, China. where $m$ is the number of objective functions; $\mathbf{x}=$ $\left(x_{1}, \ldots, x_{n}\right)^{T}$ is an $n$-dimensional decision vector; $n$ is the number of decision variables; $\mathrm{x} \in \mathbb{S}$, and $\mathbb{S} \subseteq \mathbb{R}^{n}$ denotes the search space. $g_{j}(\mathbf{x})$ and $h_{j}(\mathbf{x})$ are the $j$-th inequality and equality constraints, respectively. When $m>3$, Equation (1) is a constrained many-objective optimization problem (CMaOP) [4]. In real-world applications, there are many problems that can be formulated as a $\mathrm{CMaOP}$, such as manyobjective multi-cast routing problems [5], many-objective software product line problems [6], interval many-objective optimization problems [7] and many-objective vehicle routing problems [8].

For a CMOP/CMaOP, the degree of constraint violation $\left(\mathrm{CV}_{j}\right)$ of $\mathbf{x}$ at the $j$-th constraint is calculated as:

$$
\mathrm{CV}_{j}(\mathbf{x})=\left\{\begin{array}{ll}
\max \left(0, g_{j}(\mathbf{x})\right), & j=1, \cdots, p \\
\max \left(0,\left|h_{j}(\mathbf{x})\right|-\eta\right), & j=p+1, \cdots, q
\end{array},\right.
$$

where $\eta$ is a very small numerical quantity to relax the equality constraints into inequality ones. The value $10^{-4}$ is adopted in this work for the C-DTLZ and DC-DTLZ problems; however, other values could be used depending on the application domain. Finally, the overall constraint violation $(\mathrm{CV})$ of $\mathrm{x}$ is calculated as:

$$
\mathrm{CV}(\mathbf{x})=\sum_{j=1}^{q} \mathrm{CV}_{j}(\mathbf{x}) .
$$

The solution $\mathbf{x}$ is feasible if $\mathrm{CV}(\mathbf{x})=0$; otherwise, it is infeasible.

Due to the success of using evolutionary algorithms (EAs) for constrained single-objective optimization [9], constrained multi-objective optimization [10], and many-objective optimization [11]-[14], the use of EAs for CMaOPs is possible and interesting. However, there are some challenges as follows:

- Besides the optimization strategies for different objective functions in high-dimensional objective space, efficient constraint-handling techniques (CHTs), such as penalty function [15] and stochastic ranking [16], are required to minimize the $\mathrm{CV}$ to handle the constraints.

- The infeasibility caused by constraints may result in different shapes of constrained Pareto fronts (CPFs), increasing the difficulty faced by constrained many-objective optimization evolutionary algorithms (CMaOEAs). Therefore, achieving a good balance among feasibility, conver- 
gence, and diversity during the optimization process is essential to handle different types of CPFs in CMaOPs [17].

- The Pareto dominance pressure dramatically decreases when there is an increase of the objective space dimensionality [18], [19], and this problem becomes harder to solve with the presence of infeasibility constraints. Therefore, the strengthened dominance is crucial to strengthen the selection pressure for CMaOPs.

In the evolutionary computation (EC) community, research efforts on the use of EAs for solving the CMaOPs is lukewarm [20].Although there are many existing techniques for $\mathrm{CMOPs} / \mathrm{CMaOPs}$, the efficiency of balancing convergence, diversity and feasibility of these approaches is still insufficient. There is much room for improving the performance of EAs to effectively deal with the CMaOPs. Based on this consideration, in this paper, we propose a novel CMaOEA with enhanced mating and environmental selections. The proposed method is referred to as CMME. Its main features include: i) two ranking strategies are proposed for feasibility, convergence and diversity promotion; ii) an individual density estimation technique is designed and the crowding distance [21] is used to improve diversity; iii) $\theta$-dominance [22] is applied to enhance the selection pressure on convergence and diversity; and iv) techniques i-iii are combined into the enhanced mating and environmental selections to achieve a good balance of feasibility, convergence, and diversity to effectively solve CMaOPs.

The main contributions are as follows:

1) In the enhanced mating selection, a "Type 1 Ranking" $\left(\mathrm{R}^{\# 1}\right)$ strategy that prefers feasible or nondominated solutions is proposed for the promotion of feasibility, convergence and diversity. The feasible solutions are preferred for promotion of feasibility, and the nondominated solutions, without considering any constraint, are preferred for promotion of convergence. Meanwhile, the designed individual density estimation strategy, which prefers solutions located in sparse areas, is applied for the promotion of diversity.

2) In the enhanced environmental selection, a "Type 2 Ranking" $\left(\mathrm{R}^{\# 2}\right)$ strategy, which prefers feasible and nondominated solutions is proposed to promote convergence and feasibility. In addition, the crowding distance and $\theta$-dominance are also used in the environmental selection to improve diversity and convergence.

To extensively evaluate the performance of the proposed CMME algorithm, $10 \mathrm{CMaOPs}$ with a variable number of objective functions, six three-objective CMOPs and three realworld application CMOPs were chosen as the test suite. CMME was compared with nine related methods. The results indicate the superiority of CMME for solving different CMOPs/CMaOPs.

The remainder of this paper is organized as follows. Section II introduces the background and related work. In Section III, the proposed CMME method is proposed in detail, followed by a presentation of the experiments and analysis in Section IV. Finally, Section V presents the conclusions and points out some possible future directions.

\section{BACKGROUND AND RELATED WORK}

This section first describes the background, including some representative CHTs and the $\theta$-dominance. Then, related CMaOEAs are briefly reviewed.

\section{A. Representatives of Constraint-Handling Techniques}

1) Constrained Dominance Principle: Inspired by the feasibility-rule-based CHT [23], Deb et al. [21] developed the constrained dominance principle (CDP) for CMOPs. Specifically, solution $\mathbf{x}$ is said to constraint dominate $\mathbf{y}$ (denoted as $\mathbf{x} \prec_{C D P} \mathbf{y}$ ), if any of the following situations is satisfied:

- $\mathbf{x}$ is feasible, while $\mathbf{y}$ is not;

- Both $\mathbf{x}$ and $\mathbf{y}$ are feasible, and $\mathbf{x} \prec \mathbf{y}$;

- $\mathbf{x}$ and $\mathbf{y}$ are infeasible, and $\mathrm{CV}(\mathbf{x})<\mathrm{CV}(\mathbf{y})$.

Here $\mathbf{x} \prec \mathbf{y}$ indicates $\mathbf{x}$ dominates $\mathbf{y}$ based on the traditional Pareto dominance. Due to its simple definition and light implementation, CDP is widely used in various CMOEAs, such as NSGA-II [21] and C-MOEA/D [24]. Nevertheless, CDP prefers feasible solutions over infeasible ones [25], [26], which always results in premature convergence, especially when the feasible regions are discontinuous or faulted.

2) $\varepsilon$-constrained Technique: To overcome the shortage of CDP, a relaxation factor $\varepsilon$ is considered and $\varepsilon$-constrained technique has been designed [27]. For two solutions $\mathbf{x}$ and $\mathbf{y}$, the $\varepsilon$-constrained dominance $\mathbf{x} \prec_{\varepsilon} \mathbf{y}$ holds if one of the following conditions is satisfied:

- $\phi(\mathbf{x})<\varepsilon, \phi(\mathbf{y})<\varepsilon, \mathbf{x} \prec \mathbf{y}$;

- $\phi(\mathbf{x})=\phi(\mathbf{y})=\varepsilon, \mathbf{x} \prec \mathbf{y}$;

- $\phi(\mathbf{x})>\varepsilon, \phi(\mathbf{y})>\varepsilon, \phi(\mathbf{x})<\phi(\mathbf{y})$.

Due to the existence of the relaxation factor $\varepsilon$, infeasible solutions are likely to be selected; thus, the exploration ability is enhanced. In recent years, $\varepsilon$-constrained technique has been widely used [28]-[31]. However, although is performs well on CMOPs with large infeasible regions, $\varepsilon$-constrained technique also leads to the instability of search preference. An $\varepsilon$ that is too large favours the selection of infeasible solutions far from feasible regions, which degenerates the search process.

3) Penalty Function: Another possible way to alleviate the shortage of CDP is by using the penalty function [15]. Generally, the fitness $\mathbf{F}^{\prime}(\mathbf{x})$ of a solution $\mathbf{x}$ based on the penalty function is calculated as

$$
\mathbf{F}^{\prime}(\mathbf{x})=\mathbf{F}(\mathbf{x})+\beta \phi(\mathbf{x}),
$$

where $\beta$ is the penalty factor. Ever since the proposal of penalty function, it has received a lot of attention [32], [33]. However, the adaption of parameter $\beta$ to fit different kinds of problems is still challenging [25].

4) Two-rankings-based Fitness: Ma et al. [25] developed a two-rankings-based fitness (TRF). Two rankings $R_{c}(\mathbf{x})$ and $R_{p}(\mathbf{x})$ for a solution $\mathbf{x}$ are defined, where $R_{c}(\mathbf{x})$ and $R_{p}(\mathbf{x})$ are the rankings of $\mathrm{x}$ based on CDP and the traditional Pareto dominance, respectively. Note that, in $R_{p}(\mathbf{x})$, the constraints are not considered. Based on these two rankings, the weighted sum fitness of $\mathbf{x}$ is calculated as:

$$
\min \hat{F}(\mathbf{x})=\alpha R_{c}(\mathbf{x})+(1-\alpha) R_{p}(\mathbf{x}),
$$


and

$$
\alpha=0.5+0.5 P_{f},
$$

where $P_{f}$ is the proportion of feasible solutions in the current population, which adaptively reflects the evolution status. It is used to achieve a balance between constraints and objective function values. But actually, if the population mainly consists of feasible solutions $(\alpha \approx 1)$, then TRF has the same effect as CDP. In this situation, infeasible solutions are very likely to be rejected; thus, jumping out of the local feasible region becomes hard. Therefore, when dealing with some convergence-hard or diversity-hard CMOPs [4], TRF will fall into the same dilemma (trapped in local optimal) as CDP does.

5) Two-archive Technique: $\mathrm{Li}$ et al. [10] introduced a constrained two-archive EA (C-TAEA) for CMOPs and CMaOPs, where the convergence, feasibility, and diversity are balanced through the two-archive technique (TAT). In TAT, two archives ( i.e. convergence archive (CA) and diversity archive (DA)) co-evolve simultaneously. The archive CA is used to promote the convergence and feasibility, while DA is used to enhance diversity. Experimental results showed that the CTAEA can handle CMOPs and CMaOPs with intersected and discontinuous feasible regions [10]. However, as pointed out in [26], its mating selection strategy usually chooses one parent individual from CA and the other from DA. The generated offspring will locate in the middle region between CA and DA; thus, neither possessing better convergence, feasibility than individuals in CA, nor being able to promote the diversity of DA. In other words, the generated offspring contain much useless information.

6) Two-stage Technique: Some researchers have applied the two-stage strategy for constraint handling. ToP [34] deals with CMOPs by separating the search process into two phases. In the first phase, a reference vector is used to transform the original CMOP into a constrained single objective optimization problem. Then in the second phase, the original CMOP is optimized. CMOEA-MS [35] adopts two adaptively switched stages. One solves the original CMOP, the other ignores all constraints to solve the unconstrained MOP. PPS [36] includes push stage and pull stage. The push stage ignores all constraints to push the population near the PF, then the pull stage considers all constraints to pull the population near the CPF.

\section{B. Strengthened Dominance: $\theta$-dominance}

To enhance the selection pressure for solving manyobjective optimization problems (MaOPs), Yuan et al. proposed a strengthened dominance, namely $\theta$-dominance [22]. The $\theta$-dominance is mainly based on the penalty boundary intersection (PBI) [37] and multi-objective to multi-objective (M2M) [38] techniques.

1) PBI: In MOEA/D, Zhang and Li [37] developed the PBI distance measure for decomposition-based approach. Let $\mathbf{x}$ be a solution, and $\mathbf{f}(\mathbf{x})$ is the vector of the objective functions. The objective values are first normalized as:

$$
\bar{f}_{i}(\mathbf{x})=\frac{f_{i}(\mathbf{x})-z_{i}^{*}}{z_{i}^{\text {nad }}-z_{i}^{*}}
$$

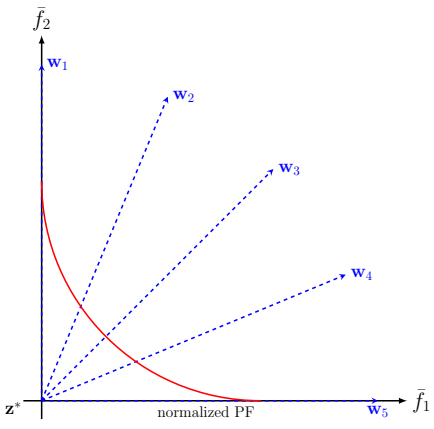

Fig. 1. Illustration of the subregion division with M2M.

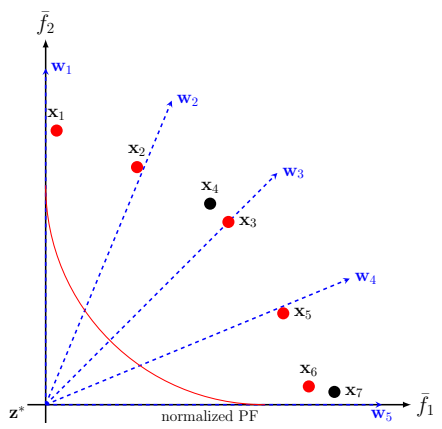

Fig. 2. Illustration of the $\theta$-dominance principle, where the red points are better than the black ones based on $\theta$-dominance.

where $i=1, \cdots, m ; \mathbf{z}^{*}$ consists of $\min _{\mathbf{x} \in \mathbb{S}} f_{i}(\mathbf{x})$, and $\mathbf{z}^{\text {nad }}$ consists of $\max _{\mathbf{x} \in \mathbb{S}} f_{i}(\mathbf{x})$.

Let $\mathbf{w}$ be the reference vector of $\mathbf{x} ; d_{1}(\mathbf{x})$ be the projection distance, and $d_{2}(\mathbf{x})$ be the vertical distance. $d_{1}(\mathbf{x})$ and $d_{2}(\mathbf{x})$ can be formulated as:

$$
\begin{gathered}
d_{1}(\mathbf{x})=\frac{\left\|\overline{\mathbf{f}}(\mathbf{x})^{T} \mathbf{w}\right\|}{\|\mathbf{w}\|} . \\
d_{2}(\mathbf{x})=\left\|\overline{\mathbf{f}}(\mathbf{x})-d_{1}(\mathbf{x}) \frac{\mathbf{w}}{\|\mathbf{w}\|}\right\| .
\end{gathered}
$$

Then PBI is calculated as:

$$
\operatorname{PBI}(\mathbf{x})=d_{1}(\mathbf{x})+\theta d_{2}(\mathbf{x})
$$

where $\theta>0$ is a predefined penalty factor, which is usually set to be 5 .

2) $M 2 M$ : To improve the performance of MOEA/D on problems with an extreme bias, Liu et al. [38] developed the M2M technique, which decomposes the objective space into a number of simple subregions. In M2M, each subregion has its own sub-population. Based on M2M, the density of each subregion can be evaluated to reflect the crowding degree of each sub-population. Figure 1 shows the subregion division with M2M, where the objective space is divided into different subregions by the reference vectors.

3) $\theta$-dominance: Based on PBI and M2M, in $\theta$-DEA [22], the $\theta$-dominance was presented and defined as follows:

Definition 1. Solution $\mathrm{x}$ is said to $\theta$-dominate solution $\mathrm{y}$, denoted as $\mathbf{x} \prec \theta \mathbf{y}$, if both $\mathbf{x}$ and $\mathbf{y}$ are in the same subregion, and $\mathrm{PBI}(\mathbf{x})<\mathrm{PBI}(\mathbf{y})$. 
In Figure 2, the $\theta$-dominance principle is illustrated. There are five reference vectors $\left(\mathbf{w}_{1}, \cdots, \mathbf{w}_{5}\right)$ and seven individuals $\left(\mathbf{x}_{1}, \cdots, \mathbf{x}_{7}\right)$. Based on M2M, $\mathbf{x}_{1}, \mathbf{x}_{2}$, and $\mathbf{x}_{5}$ are associated with $\mathbf{w}_{1}, \mathbf{w}_{2}$, and $\mathbf{w}_{4}$, respectively; $\mathbf{x}_{3}$ and $\mathbf{x}_{4}$ are associated with $\mathbf{w}_{3} ; \mathbf{x}_{6}$ and $\mathbf{x}_{7}$ are associated with $\mathbf{w}_{5}$. Then, based on the $\theta$-dominance, $\mathbf{x}_{1}, \mathbf{x}_{2}, \mathbf{x}_{3}, \mathbf{x}_{5}$, and $\mathbf{x}_{6}$ are assigned to the first front, while $\mathbf{x}_{4}$ and $\mathbf{x}_{7}$ are in the second front. Therefore, combining the advantages of M2M and PBI, the $\theta$-dominance can provide the strengthened selection pressure on both convergence and diversity [22].

\section{Existing CMaOEAs}

This section briefly reviews some representative related CMaOEAs.

Combined with NSGA-II [21], Saxena et al. [20] presented an EA with $\varepsilon$-dominance, controlled infeasibility, and a nonlinear dimensionality reduction scheme for CMaOPs. Jain and Deb et al. [24] embedded the CDP [21] into NSGAIII [39] and MOEA/D [37] to extend them for CMaOPs. In I-DBEA [40], an adaptive $\varepsilon$ formulation was designed to handle the constraints. Cooperating with a preemptive distance comparison scheme and two independent distance measures, IDBEA is capable of dealing with CMaOPs. Elarbi et al. [41], [42] introduced an isolated solution-based constrained Pareto dominance, which favors infeasible solutions associated with isolated sub-regions or with a smaller CV to exploit infeasible isolated solutions. Miyakawa et al. [43] proposed the CMOEA/D-DMA for CMaOPs, where the directed mating and archives of infeasible solutions are used. Li et al. [10] introduced the C-TAEA with TAT to balance convergence, feasibility, and diversity to deal with CMaOPs. In CCMODE [44], a co-evolutionary framework with $(m+1)$ subpopulations uses $m$ sub-populations for constrained singleobjective optimization and an archive sub-population for constrained $m$-objective optimization. Zhou et al. [45] designed a tri-goal evolution framework, where three indicators for convergence, diversity, and feasibility are presented for CMaOPs. Fan et al. [31] used a pull and push search (PPS) framework with two-stage strategy, which handles the constrained and unconstrained MaOPs separately to accelerate the approach to the CPF. Wang and $\mathrm{Xu}$ [46] developed an angle-based EA with infeasibility information, in which different kinds of infeasible solutions are utilized in the environmental and mating selections. Jiao et al. [28] transformed CMaOP into a dynamic CMaOP with the $\epsilon$-CDP for handling constraints and optimizing objectives simultaneously.

The features and drawbacks of these existing CMaOEAs are shown in Table I.

\section{OUR APPROACH: CMME}

In this section, the proposed method is elucidated, including the motivation, individual density estimation, two ranking strategies, enhanced mating and environmental selections, framework, remarks and complexity.
TABLE I

THE MAIN FEATURES AND DRAWBACKS OF EXISTING CMAOEAS

\begin{tabular}{|l|l|l|l|}
\hline \hline Algorithm & Type & CHT & Drawbacks \\
\hline NSGA-III & $\begin{array}{l}\text { dominance } \\
\text { decomposition }\end{array}$ & CDP & $\begin{array}{l}\text { easily trapped in } \\
\text { local feasible region }\end{array}$ \\
\hline DCNSGA-III & $\begin{array}{l}\text { dominance } \\
\text { decomposition }\end{array}$ & $\varepsilon$-constrained & adaption of $\varepsilon$ \\
\hline PPS & decomposition & $\varepsilon$-constrained & adaption of $\varepsilon$ \\
\hline I-DBEA & decomposition & $\varepsilon$-constrained & adaption of $\varepsilon$ \\
\hline C-TAEA & $\begin{array}{l}\text { dominance } \\
\text { decomposition }\end{array}$ & CDP \& TAT & $\begin{array}{l}\text { inappropriate mating strategy \& } \\
\text { easily trapped in local feasible region }\end{array}$ \\
\hline TiGE-2 & dominance based & CDP \& TRF & $\begin{array}{l}\text { existence of infeasible solutions } \\
\text { in final solution set \& } \\
\text { easily trapped in local feasible region }\end{array}$ \\
\hline
\end{tabular}

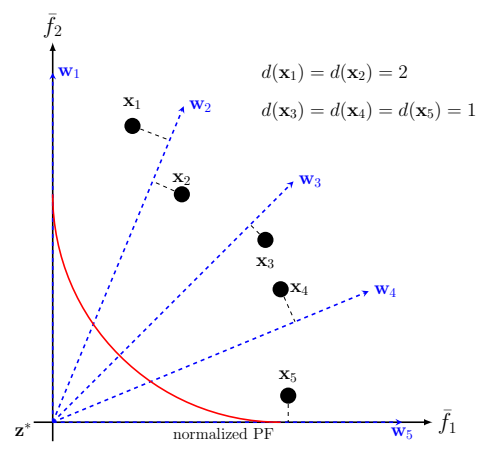

Fig. 3. Illustration of the proposed individual density estimation, where $d\left(\mathbf{x}_{i}\right)$ represents the density of $\mathbf{x}_{i}$

\section{A. Motivation}

1) Research Gap in CMaOEA: Unlike the many research outcomes of MaOEAs in the EC community [11], much less research has been done on CMaOPs. There is still much room to improve the performance of EAs for dealing with CMaOPs.

Balancing feasibility, convergence, and diversity is the main challenge for handling CMaOPs. To effectively solve CMaOPs, one possible way is to design new enhanced mating and environmental selections [47] which can achieve the balance of feasibility, convergence, and diversity. Based on this consideration, we propose a novel CMaOEA with enhanced mating and environmental selections to achieve a good balance of feasibility, convergence, and diversity.

2) Expanding $\theta$-dominance to $C M a O E A$ : Experimental results show that $\theta$-dominance is effective in handling MaOPs. With the help of reference vectors and the strengthened dominance relation, $\theta$-DEA, which applies $\theta$-dominance, performs well on balancing convergence and diversity in dealing with MaOPs. However, it can not solve CMaOPs without a CHT to handle constraints. Therefore, expanding the effective $\theta$ dominance to CMaOEA is worth trying.

3) Multi-ranking for Balancing Convergence, Diversity and Feasibility: As mentioned in Section II-A and Table I, the existing CHTs, as well as the existing CMaOEAs, still have some shortages that must be overcome. Inspired by the idea of TRF and TAT, we use a multi-ranking strategy to achieve a balance of different goals. Further, Pareto dominance has a preference for well-converged solutions, while the $\mathrm{CV}$ value represents the feasibility performance. Since the core issue of CMaOEA is achieving balance between convergence, diversity and feasibility, designing different rankings for different goals 

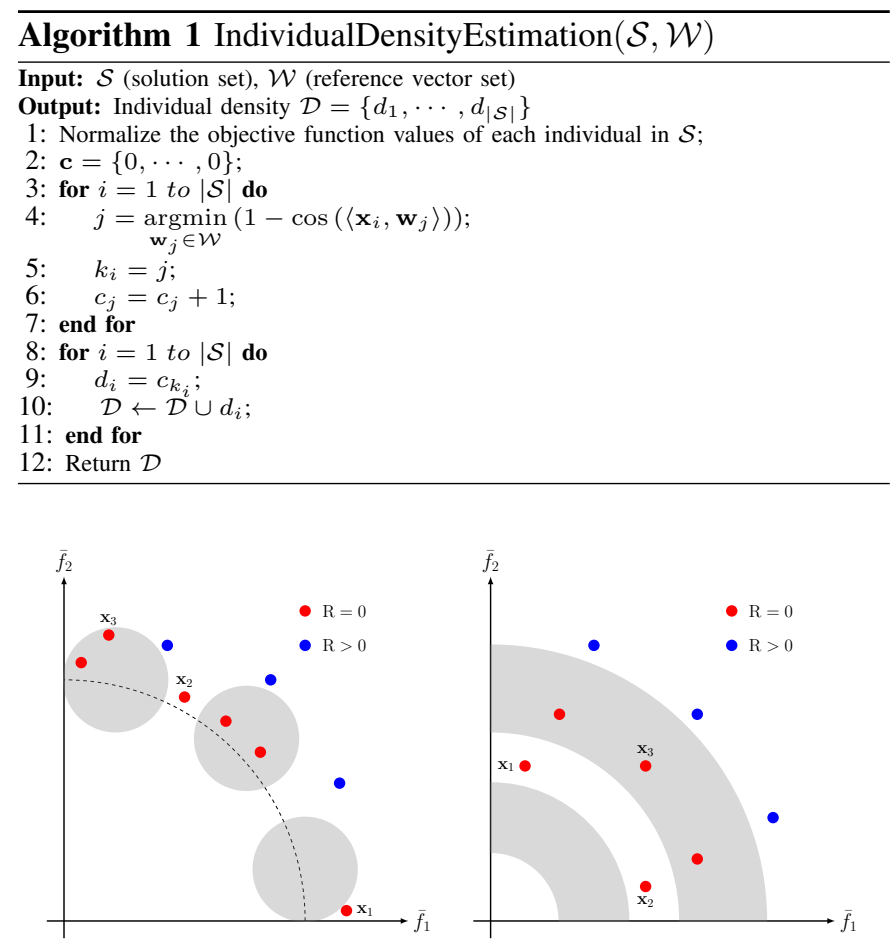

(a) Illustration of applying $\mathrm{R}^{\# 1}$ on (b) Illustration of applying $\mathrm{R}^{\# 1}$ on discontinuous feasible regions. faulted feasible regions.

Fig. 4. Illustration of $R^{\# 1}$ used in the mating selection.

is promising. Also, the application of different rankings in mating and environmental selection is promising.

\section{B. Individual Density Estimation}

Based on M2M, the objective space can be divided into different subregions. To promote the diversity of the solution set, the individual density estimation is proposed as shown in Algorithm 1. First, the objective function values of each individual in the solution set $\mathcal{S}$ are normalized by using Equation (6) (line 1). Also the $N$ subregion density index is initialized, which is the same as the size of the reference vector set (line 2). Then, each individual is associated with its nearest reference vector based on the cosine similarity (line 4). The individual density is estimated by calculating the number of individuals in this individual's located subregion. Note that the constraints are not considered when estimating the individual density.

To better understand the principle of Algorithm 1, Figure 3 gives an example for the individual density estimation according to M2M. In Figure 3, the objective space is divided into five subregions based on the five reference vectors (i.e., $\left.\mathbf{w}_{1}, \cdots, \mathbf{w}_{5}\right)$. Based on the cosine similarity between the individual and its nearest reference vector, each individual is associated with the corresponding reference vector. Then, the density of the individual is represented by the number of individuals in its located subregion. In this way, $d\left(\mathbf{x}_{1}\right)=$ $d\left(\mathbf{x}_{2}\right)=2, d\left(\mathbf{x}_{3}\right)=d\left(\mathbf{x}_{4}\right)=d\left(\mathbf{x}_{5}\right)=1$, where $d\left(\mathbf{x}_{i}\right)$ indicates the density of $\mathbf{x}_{i}$.
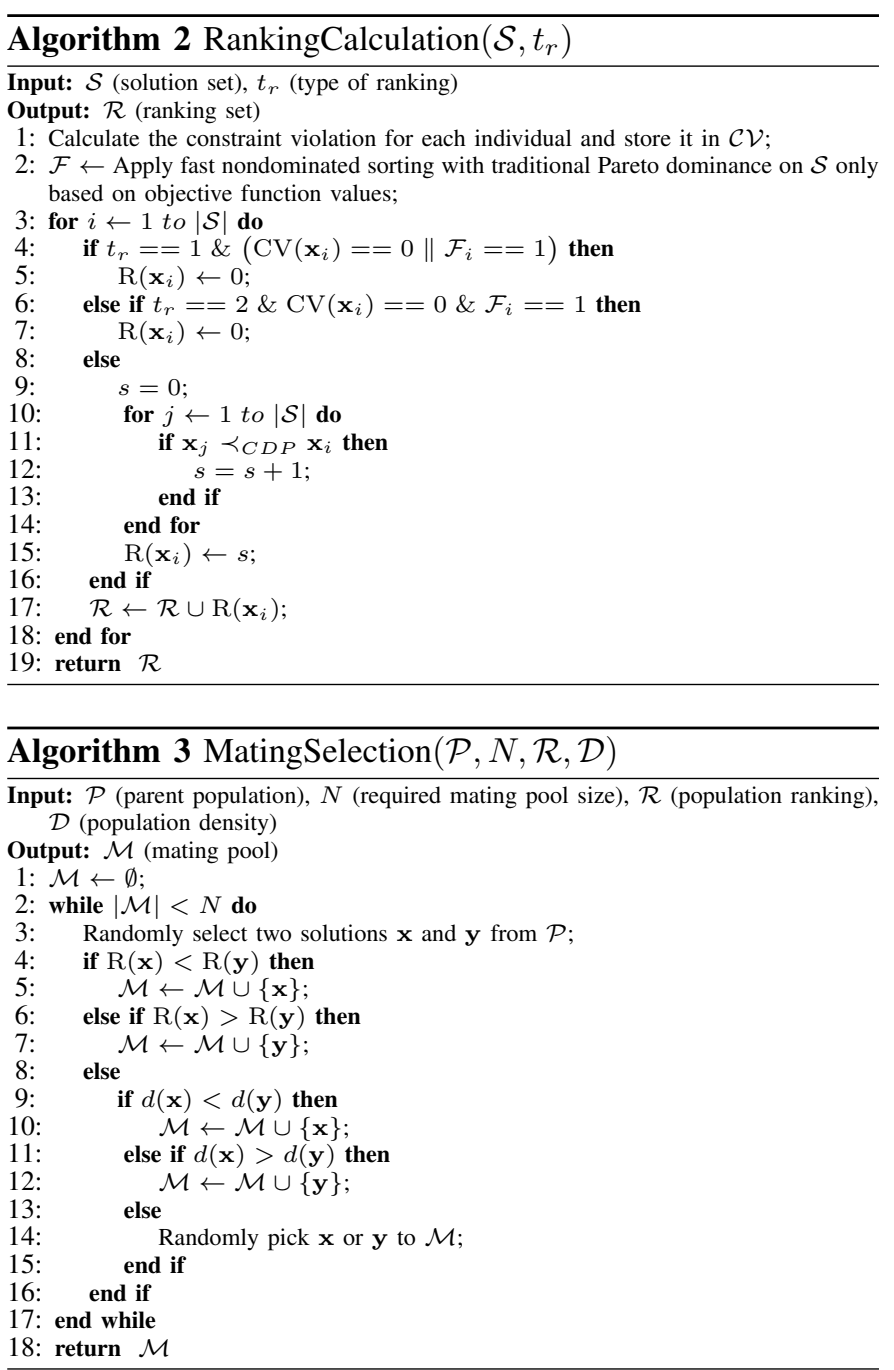

\section{Enhanced Mating Selection}

To effectively solve CMaOPs, besides convergence and diversity, feasibility is very important. In the mating selection, one of the main goals is to detect new feasible areas. Therefore, it is essential to select suitable parents to form the mating pool $\mathcal{M}$ for offspring generation. Based on this consideration, we propose the $\mathrm{R}^{\# 1}$ strategy to assign the rank of an individual to measure its suitability.

1) Type 1 Ranking $\left(\mathrm{R}^{\# 1}\right)$ : The $\mathrm{R}^{\# 1}$ rank of an individual $\mathbf{x}$ is defined as:

Definition 2. $\mathrm{R}_{1}(\mathrm{x})=0$, if $\mathrm{x}$ is feasible or it is a nondominated individual; otherwise, $\mathrm{R}_{1}(\mathrm{x})$ is equal to the number of individuals that dominate $\mathbf{x}$ according to CDP [21].

According to Definition 2, all feasible individuals and all nondominated individuals are assigned the best ranks. They have more chance to be selected into $\mathcal{M}$. The pseudo code of ranking calculation is shown in Algorithm 2. First, the constraint violation and dominance front number of $\mathcal{S}$ are calculated and stored in $\mathcal{C V}$ and $\mathcal{F}$ (lines 1-2), respectively. Then, the rank of each individual in $\mathcal{S}$ is calculated according to a specific ranking strategy. $t_{r}$ is a flag to determine which type of ranking strategy is used. 

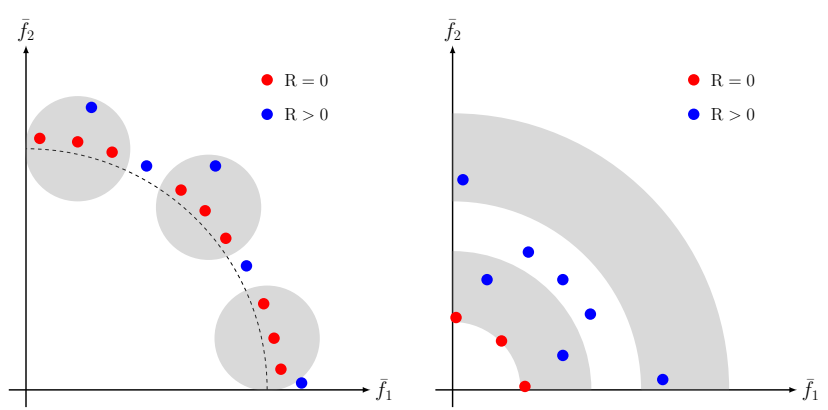

(a) Illustration of applying $\mathrm{R}^{\# 2}$ on (b) Illustration of applying $\mathrm{R}^{\# 2}$ on discontinous feasible regions. faulted feasible regions.

Fig. 5. Illustration of $\mathrm{R}^{\# 2}$ used in the environmental selection.

As depicted in Figure 4, although $\mathbf{x}_{1}$ and $\mathbf{x}_{2}$ are infeasible solutions, they are ranked 0 because they are in the first dominance front. Their inclusion in $\mathcal{M}$ could guide the search toward undeveloped feasible regions. $\mathbf{x}_{3}$ is not in the first front; however, it is also ranked 0 because of its feasibility. The inclusion of $\mathbf{x}_{3}$ will promote the exploitation of developed feasible regions.

2) Mating Selection with $\mathrm{R}^{\# 1}$ : Combined with $\mathrm{R}^{\# 1}$, the enhanced mating selection is given in Algorithm 3. It is based on the tournament selection. First, two randomly selected individuals $\mathbf{x}$ and $\mathbf{y}$ are compared based on their ranks and the better one is added into $\mathcal{M}$. If both of them have the same ranks, the one with smaller density is added into $\mathcal{M}$. If $\mathrm{R}(\mathbf{x})=\mathrm{R}(\mathbf{y})$ and $d(\mathbf{x})=d(\mathbf{y})$, then either $\mathbf{x}$ or $\mathbf{y}$ is randomly chosen for $\mathcal{M}$. The above procedure continues until $|\mathcal{M}| \geq N$.

The benefit from using the $\mathrm{R}^{\# 1}$ strategy and individual density estimation is that the individuals in $\mathcal{M}$ could guide the search to explore new feasible regions and exploit the existing feasible regions with diversity promotion. Therefore, the mating selection is able to make a good tradeoff between exploration and exploitation on feasibility. Note that if the population only contains feasible solutions, this situation usually appears in the late stage of evolution. The mating selection will promote convergence and diversity.

\section{Enhanced Environmental Selection}

1) Type 2 Ranking ( $\left.\mathrm{R}^{\# 2}\right)$ : In environmental selection, a strengthened selection pressure is required to balance feasibility, convergence, and diversity to obtain the next parent population. Therefore, we propose the $\mathrm{R}^{\# 2}$ strategy to assign the rank of an individual $\mathrm{x}$ :

Definition 3. $\mathrm{R}_{2}(\mathrm{x})=0$, if $\mathrm{x}$ is a feasible and nondominated individual; otherwise, $\mathrm{R}_{2}(\mathrm{x})$ is equal to the number of individuals that dominate $\mathrm{x}$ according to $C D P$.

From Definition 3, the individuals are ranked 0 , if they are feasible and in the first front. As depicted in Figure 5, only the red points are assigned the best ranks. Therefore, the $\mathrm{R}^{\# 2}$ strategy prefers the individuals that are closer to the constrained true PF. It can enrich the selection pressure on the convergence and feasibility.

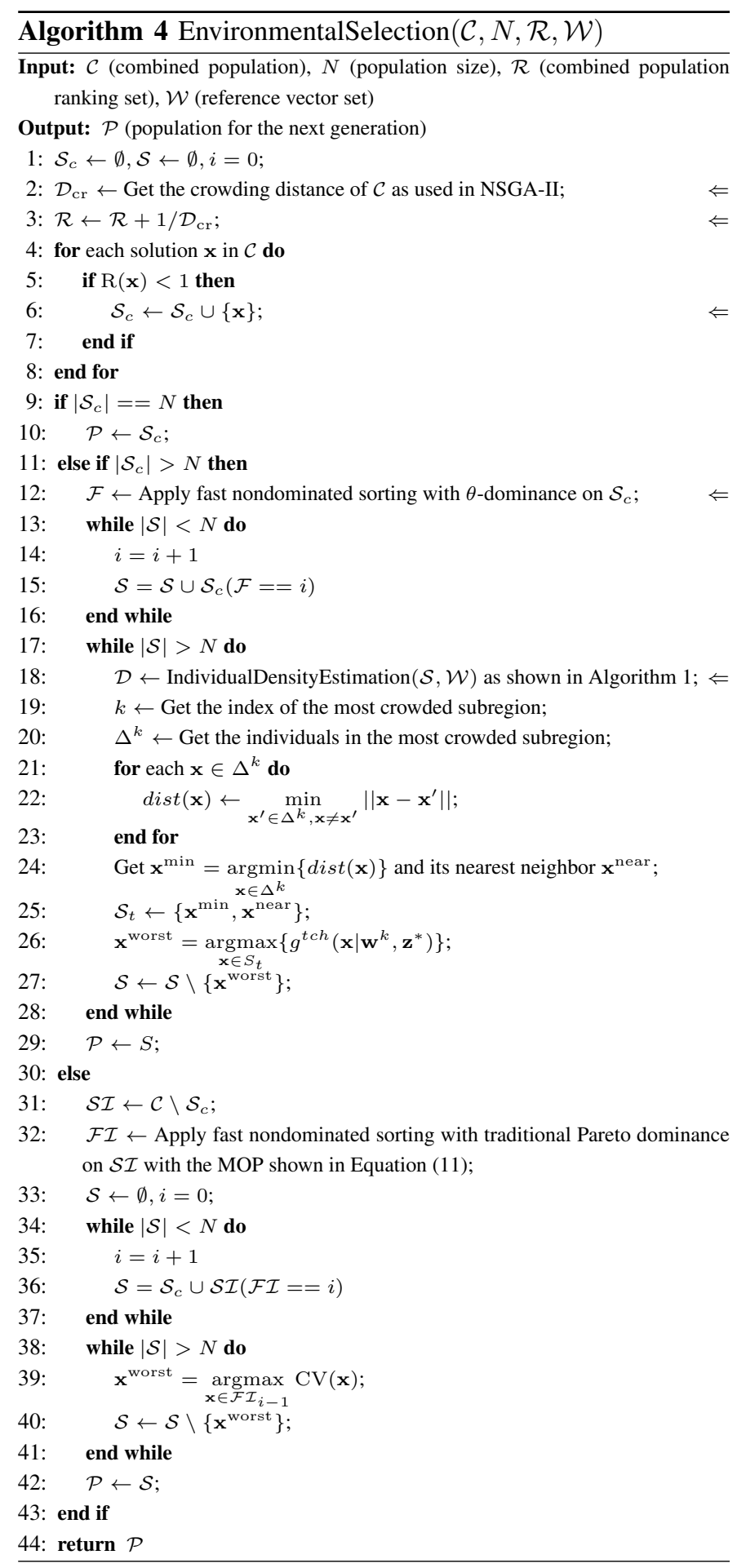

2) Environmental Selection with $\mathrm{R}^{\# 2}$ : Based on the $\mathrm{R}^{\# 2}$ strategy, the enhanced environmental selection is developed in Algorithm 4. First, the crowding distance of each individual in $\mathcal{C}$ is calculated by the technique presented in NSGA-II [21]. All crowding distance values are saved into $\mathcal{D}_{\text {cr }}$ (line 2 ). Then, the distance of each individual is combined with its rank as the supplementary diversity (line 3). Subsequently, all individuals in $\mathcal{C}$ with ranks less than 1 are selected into the candidate set $\mathcal{S}_{c}$ (lines 4-8). Based on the size of $\mathcal{S}_{c}$, there are three situations: 
1) $\left|\mathcal{S}_{c}\right|=N$. In this case, $\mathcal{S}_{c}$ can be used directly as the population for the next generation.

2) $\left|\mathcal{S}_{c}\right|>N$. This situation means that most of the individuals in $\mathcal{C}$ are feasible with good distribution. In this case, the selection strategy should be designed to promote convergence and diversity.

3) $\left|\mathcal{S}_{c}\right|<N$. On the contrary, this situation reflects that either the number of feasible solutions with good distribution is not enough, or the infeasible solutions are of the majority. Thus, the selection strategy should be inclined to the feasibility.

For $\left|\mathcal{S}_{c}\right|>N$, some poor performed feasible individuals are discarded from $\mathcal{S}_{c}$ to obtain the next population $\mathcal{P}$ with balanced convergence and diversity.

- First, $\mathcal{S}_{c}$ undergoes the fast nondominated sorting with the $\theta$-dominance to get the front $\mathcal{F}$ (line 12).

- Then, the individuals in $\mathcal{S}_{c}$ are chosen front by front and added into $\mathcal{S}$ until $|\mathcal{S}| \geq N$ (lines 13-16).

- Subsequently, the worst individual is deleted one by one until $|\mathcal{S}|=N$ (lines 17-28). In line 18, the individual density $\mathcal{D}$ of $\mathcal{S}$ is estimated. In lines 19-20, the index $k$ of the most crowded subregion is detected based on the individual densities, and the individuals in this subregion are saved in $\Delta^{k}$. In lines 21-23, for each individual $\mathbf{x} \in \Delta^{k}$, its minimal distance $\operatorname{dist}(\mathbf{x})$ is calculated. In lines 24-25, in $\Delta^{k}$, the individual $\mathrm{x}^{\mathrm{min}}$ with the minimal $\operatorname{dist}(\mathbf{x})$ and its nearest neighbor $\mathbf{x}^{\text {near }}$ are found and saved in $\mathcal{S}_{t}$. For individuals in $\mathcal{S}_{t}$, the one with greater Tchebycheff distance (i.e., with worse convergence) is obtained (line 26) and deleted (line 27). The Tchebycheff distance can be mathematically formulated as

$$
g^{t c h}\left(\mathbf{x} \mid \mathbf{w}^{k}, \mathbf{z}^{*}\right)=\max _{1 \leq j \leq m}\left\{\frac{\left|f_{j}(\mathbf{x})-\mathrm{z}_{j}^{*}\right|}{\mathrm{w}_{j}^{k}}\right\},
$$

where $\mathbf{z}^{*}$ is the ideal point; $\mathbf{w}^{k}$ is the reference vector corresponding to the most crowded subregion, and $f_{j}(\mathbf{x})$ represents the $j$-th objective value of $\mathbf{x}$.

For $\left|\mathcal{S}_{c}\right|<N$, the CA update strategy proposed in CTAEA [10] is used to explore the feasible regions.

- First, the infeasible individuals in $\mathcal{C}$ are separated and saved in $\mathcal{S I}$ in line 31.

- Then, the individuals in $\mathcal{S I}$ are evaluated by a new biobjective optimization problem formulated as

$$
\begin{array}{cl}
\text { minimize } & \mathbf{F}(\mathbf{x})=\left(F_{1}(\mathbf{x}), F_{2}(\mathbf{x})\right)^{T}, \\
\text { where } & \left\{\begin{array}{l}
F_{1}(\mathbf{x})=\mathrm{CV}(\mathbf{x}), \\
F_{2}(\mathbf{x})=g^{t c h}\left(\mathbf{x} \mid \mathbf{w}^{k}, \mathbf{z}^{*}\right) .
\end{array}\right.
\end{array}
$$

The front $\mathcal{F I}$ of $\mathcal{S I}$ is obtained based on the fast nondominated sorting with the MOP shown in Equation (11) (line 32).

- Afterward, the individuals in $\mathcal{S I}$ are added into $\mathcal{S}$ front by front until $\mathcal{S} \geq N$ (lines 34-36). Then, in lines 38-40, the individual with the worst constraint violation in $\mathcal{S}$ is deleted one by one until $\mathcal{S}=N$.

In the proposed enhanced environmental selection, the synergy of the following techniques can yield a good balance of feasibility, convergence, and diversity.

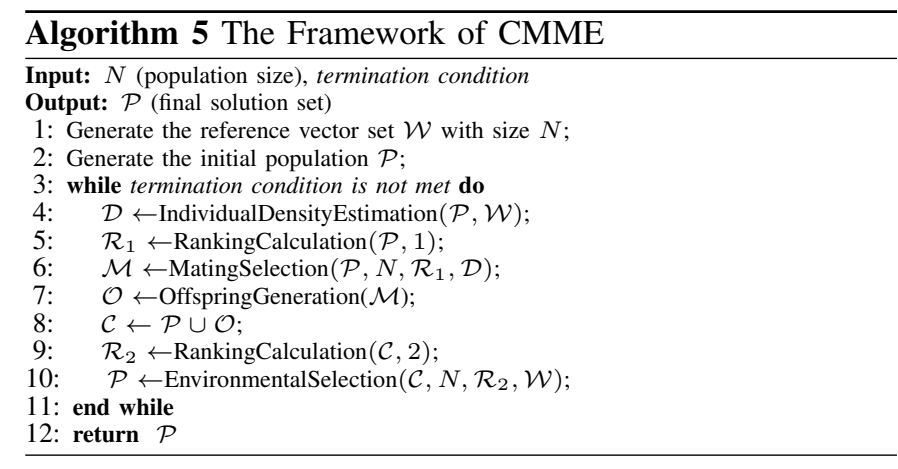

- The $\mathrm{R}^{\# 2}$ strategy is able to enhance both feasibility and convergence.

- Both the crowding distance and individual density estimation can promote diversity.

- The use of the $\theta$-dominance is capable of enhancing the selection pressure on both the convergence and diversity.

- The CA update strategy at the situation $\left|\mathcal{S}_{c}\right|<N$ in [10] can pursue the feasibility.

\section{E. Framework of CMME}

Combining the proposed procedures, the framework of the proposed CMME method is shown in Algorithm 5. Initially, the reference vector set $\mathcal{W}$ with size $N$ is randomly generated as suggested in [48], followed by the population $\mathcal{P}$ 's initialization. At each generation, the following procedures are repeated until the termination condition is met.

- In line 4 , the density of each individual in $\mathcal{P}$ is estimated as shown in Algorithm 1.

- In lines 5-6, first the individuals in $\mathcal{P}$ are ranked based on the "type 1 ranking" $\left(\mathrm{R}^{\# 1}\right)$ in Algorithm 2. Then, based on the ranking $\mathcal{R}_{1}$ and density $\mathcal{D}$, the mating pool $\mathcal{M}$ is obtained based on Algorithm 3.

- In line 7, the offspring population $\mathcal{O}$ is generated through the genetic operators. Then, in line $8, \mathcal{P}$ and $\mathcal{O}$ are combined to form the combined set $\mathcal{C}$.

- In line $9, \mathcal{C}$ is ranked based on the "type 2 ranking" $\left(\mathrm{R}^{\# 2}\right)$. And in line 10, the environmental selection procedure is performed to generate the new population $\mathcal{P}$ in Algorithm 4.

\section{F. Remarks}

- CMME includes two novel ranking mechanisms. The designed environmental and mating selection strategies are based on these two ranking strategies. Also, the individual density, crowding distance and $\theta$-dominance play essential roles. All these components work together to achieve the balance of convergence, diversity and feasibility to effectively handle CMaOPs.

- The proposed two ranking strategies are different from the TRF technique [25]. In TRF, the two rankings are used in the fitness calculation as formulated in Equation (5). However, in CMME, the $\mathrm{R}^{\# 1}$ strategy is used in the enhanced mating selection which aims to explore the undeveloped feasible regions and to exploit the existing 
feasible regions. The $\mathrm{R}^{\# 2}$ strategy is tailored for the enhanced environmental selection which aims to promote both convergence and feasibility.

- The proposed environmental selection in Algorithm 4 is similar to the CA update strategy in C-TAEA [10]. However, there are some important differences marked as " $\Leftarrow$ " in Algorithm 4: i) the CA update strategy only considers the feasibility, while in our approach the feasibility, convergence, and diversity are all taken into consideration. Based on $\mathrm{R}^{\# 2}$ ranking, the promotion of convergence and feasibility is ensured. Meanwhile the crowding distance helps with diversity promotion; ii) the traditional Pareto dominance for the CA update strategy is replaced by the $\theta$-dominance in the fast nondominated sorting to strengthen the selection pressure for the CMaOPs; and iii) individual density estimation is used in the enhanced environmental and mating selections, which has an impact on searching the sparse subregions, thus improving diversity.

- In the enhanced mating selection, the traditional Pareto dominance is used; while in the enhanced environmental selection, the $\theta$-dominance is used. The reason is that in the mating selection, feasibility is more important; however, the $\theta$-dominance may discard some feasible solutions due to its strengthened selection pressure as mentioned in Section II-B3. On the other hand, in the environmental selection, the convergence and diversity at the situation $\left|\mathcal{S}_{c}\right|>N$ are more important, and thus, the $\theta$-dominance is applied.

\section{G. Computational Complexity of CMME}

The computational complexity of CMME is composed of:

- The complexity of generating reference vector set $\mathcal{W}$ and initial population $\mathcal{P}$ are $O(m N)$ and $O(m n N)$, respectively;

- The complexity of individual density estimation in Algorithm 1 is $O\left(m|\mathcal{S}|^{2}\right)$. $|\mathcal{S}|$ usually approximates to the population size $N$, thus $O\left(m|\mathcal{S}|^{2}\right)$ can approximate to $O\left(m N^{2}\right)$;

- The complexity of calculating the ranks in Algorithm 2 is mainly caused by the fast nondominated sorting with $O\left(m N^{2}\right)[21]$

- For the mating selection shown in Algorithm 3, its complexity is $O(N)$;

- The complexity of environmental selection mainly consists of: 1) crowding distance calculation with $O(m N \log (N))$ [21]; 2) fast nondominated sorting with $O\left(m N^{2}\right)$; and 3$)$ individual density estimation with $O\left(m N^{2}\right)$.

Therefore, the overall complexity of CMME is $O\left(m N^{2}\right)$.

\section{EXPERIMENTAL RESUlts AND ANALYSis}

In this section, the performance of CMME is extensively evaluated by comparing with nine related methods on the CDTLZ [24], DC-DTLZ [10], DAS-CMOP [4], MW [17] test functions and three real-world CMOPs [49].

\section{A. Experimental Settings}

1) Benchmark Problems: 10 CMaOPs [10], [24] were chosen (i.e., C1-DTLZ1/DTLZ3, C2-DTLZ2, C3-DTLZ4, DC1-DTLZ1/DTLZ3, DC2-DTLZ1/DTLZ3, and DC3DTLZ1/DTLZ3) as the test suite. All of them are scalable, where $m=3,5,8,10$ and 15 . Additionally, the three-objective DAS-CMOP and MW instances (i.e. DAS-CMOP7 to DAS-CMOP9, MW4, MW8, MW14) were also chosen as benchmark problems for comparison. Therefore, there are totally 56 test cases. For C1-DTLZ1, DC1-DTLZ1, DC2DTLZ1, and DC3-DTLZ1, $n=m+4$, while for C1-DTLZ3, C2-DTLZ2, C3-DTLZ4, DC1-DTLZ3, DC2-DTLZ3, and DC3-DTLZ3, $n=m+9$. For DAS-CMOP7 to DAS-CMOP9, $n=30$. For MW4, MW8 and MW14, $n=15$.

2) Algorithms for Comparison: To indicate the superiority of CMME, three CMOEAs (i.e., NSGA-II-ToR [25], ToP [34], and CCMO [26],) and six CMaOEAs (i.e., NSGA-III [24], I-DBEA [40], PPS [31], C-TAEA [10], TiGE-2 [45], and DCNSGA-III [28]) were selected for comparison. All of these methods were implemented on the PlatEMO [50].

TABLE II

PARAMETER SETTINGS FOR THE 10 METHODS ON DTLZ BENCHMARK, WHERE $N$ IS THE POPULATION SIZE AND $E_{\max }$ IS THE MAXIMUM NUMBER OF FUNCTION EVALUATIONS.

\begin{tabular}{|c|c|r|}
\hline \hline$m$ & $N$ & $E_{\max }$ \\
\hline 3 & 91 & 30,000 \\
\hline 5 & 126 & 50,000 \\
\hline 8 & 156 & 80,000 \\
\hline 10 & 220 & 100,000 \\
\hline 15 & 240 & 120,000 \\
\hline
\end{tabular}

3) Genetic Operators and Parameter Settings: For CMME, the simulated binary crossover (SBX) [51] and polynomial mutation (PM) [21] were used as the genetic operators with the following parameter settings:

- Crossover probability $p_{c}=1$ and distribution index $\eta_{c}=$ 20.

- Mutation probability $p_{m}=1 / n$ and distribution index $\eta_{m}=20$.

The population size $N$ and maximal number of function evaluations $E_{\max }$ for the ten methods on C-DTLZ and DCDTLZ benchmark are listed in Table II. For those threeobjective DAS-CMOP and MW instances, $E_{\max }$ was set to 500. The parameter settings for the nine methods used for comparison were are the same as suggested in their corresponding original references. All of the parameter settings in the following text remain the same unless a change is mentioned.

Each algorithm was executed over 30 independent runs on each test case. The mean and standard deviations were recorded. The KEEL software was used to calculate the statistical results [52]. The Wilcoxon test with a significance level of 0.05 and the Friedman test with the Bonferroni correction at a significance level 0.05 were adopted to perform statistical analysis on the experimental results. Additionally, according to the Wilcoxon test, we use "+", "-", and "=" to show that a result is significantly better than, significantly worse than, or statistically similar to that obtained by CMME, respectively. 
TABLE III

AVERAGE RANKINGS OF HV AND IGD BY THE FRIEDMAN TEST FOR DIFFERENT CMAOEAS.

\begin{tabular}{|r|c||c|}
\hline \hline Algorithm & Ranking (HV) & Ranking (IGD) \\
\hline NSGA-II-ToR & 8.18 & 8.98 \\
\hline ToP & 7.31 & 7.90 \\
\hline CCMO & 5.49 & 5.55 \\
\hline NSGA-III & 4.56 & 4.06 \\
\hline I-DBEA & 7.07 & 7.24 \\
\hline PPS & 4.39 & 5.10 \\
\hline C-TAEA & $\mathbf{3 . 5 9}$ & $\mathbf{3 . 3 0}$ \\
\hline TiGE-2 & 6.41 & 5.98 \\
\hline DCNSGA-III & 4.79 & 4.40 \\
\hline CMME & $\mathbf{3 . 2 1}$ & $\mathbf{2 . 4 9}$ \\
\hline \hline
\end{tabular}

4) Performance Indicators: Two widely used indicators, i.e., inverted generational distance (IGD) [53] and hypervolume (HV) [54], are chosen to compare the performance of different algorithms.

- IGD: Represents the average distance from each reference point to the nearest individual. Suppose $\mathcal{S}^{*}$ is a set of uniformly distributed points on the true PF and $\mathcal{S}$ is the solution set. The IGD value is calculated as

$$
\operatorname{IGD}\left(\mathcal{S}^{*}, \mathcal{S}\right)=\frac{\sum_{\mathbf{x} \in \mathcal{S}^{*}} d_{\mathrm{nn}}(\mathbf{x}, \mathcal{S})}{\left|\mathcal{S}^{*}\right|},
$$

where $d_{\mathrm{nn}}(\mathbf{x}, \mathcal{S})$ is the Euclidean distance between $\mathbf{x}$ and its nearest neighbor in $\mathcal{S}$. The smaller the IGD value, the better the performance of an algorithm.

- $H V$ : Measures the volume or hypervolume of the objective space enclosed by the obtained solution set and the predefined reference point $\mathbf{z}^{r}$; HV of a solution set $\mathcal{S}$ is formulated as

$$
\mathrm{HV}(\mathcal{S})=\operatorname{VOL}\left(\bigcup\left[z_{1}, z_{1}^{r}\right] \times \cdots, \times\left[z_{m}, z_{m}^{r}\right]\right),
$$

where VOL indicates the Lebesgue measure. The algorithm with a larger $\mathrm{HV}$ value indicates better performance.

Following the approach in [26], 10,000 uniformly distributed points were sampled on the true PF for the IGD calculation. For the HV calculation, the objective values were first normalized, and in the normalized objective space, $(1.1,1.1, \cdots, 1.1)$ was adopted as the reference point.

TABLE IV

SUMMARY OF THE WiLCOXON TEST ON THE HV METRIC. “•” MEANS THE METHOD IN THE ROW IMPROVES THE METHOD OF THE COLUMN. "O” INDICATES THE METHOD IN THE COLUMN IMPROVES THE METHOD OF THE ROW. UPPER DIAGONAL OF LEVEL SIGNIFICANCE $\alpha=0.9$, AND LOWER DIAGONAL LEVEL OF SIGNIFICANCE $\alpha=0.95$.

\begin{tabular}{|r|c|c|c|c|c|c|c|c|c|c|}
\hline \hline Algorithm & $(1)$ & $(2)$ & $(3)$ & $(4)$ & $(5)$ & $(6)$ & $(7)$ & $(8)$ & $(9)$ & $(10)$ \\
\hline NSGA-II-ToR (1) & - & $\circ$ & $\circ$ & $\circ$ & $\circ$ & $\circ$ & $\circ$ & $\circ$ & $\circ$ & $\circ$ \\
\hline ToP (2) & $\bullet$ & - & $\circ$ & $\circ$ & $\circ$ & $\circ$ & $\circ$ & $\circ$ & $\circ$ & $\circ$ \\
\hline CCMO (3) & $\bullet$ & $\bullet$ & - & & & & $\circ$ & & $\circ$ & $\circ$ \\
\hline NSGA-III (4) & $\bullet$ & $\bullet$ & & - & $\bullet$ & & $\circ$ & $\bullet$ & $\bullet$ & $\circ$ \\
\hline I-DBEA (5) & $\bullet$ & $\bullet$ & & $\circ$ & - & $\circ$ & $\circ$ & $\circ$ & $\circ$ & $\circ$ \\
\hline PPS (6) & $\bullet$ & $\bullet$ & & & $\bullet$ & - & & $\bullet$ & & $\circ$ \\
\hline C-TAEA (7) & $\bullet$ & $\bullet$ & $\bullet$ & $\bullet$ & $\bullet$ & & - & $\bullet$ & $\bullet$ & \\
\hline TiGE-2 (8) & $\bullet$ & $\bullet$ & & $\circ$ & $\bullet$ & $\circ$ & $\circ$ & - & $\circ$ & $\circ$ \\
\hline DCNSGA-III (9) & $\bullet$ & $\bullet$ & & & $\bullet$ & & $\circ$ & $\bullet$ & - & $\circ$ \\
\hline CMME (10) & $\bullet$ & $\bullet$ & $\bullet$ & $\bullet$ & $\bullet$ & $\bullet$ & & $\bullet$ & $\bullet$ & - \\
\hline \hline
\end{tabular}

TABLE V

SUMMARY OF THE WILCOXON TEST ON THE IGD METRIC. "•" MEANS THE METHOD IN THE ROW IMPROVES THE METHOD OF THE COLUMN. "O” INDICATES THE METHOD IN THE COLUMN IMPROVES THE METHOD OF THE ROW. UPPER DIAGONAL OF LEVEL SIGNIFICANCE $\alpha=0.9$, AND LOWER DIAGONAL LEVEL OF SIGNIFICANCE $\alpha=0.95$.

\begin{tabular}{|r|c|c|c|c|c|c|c|c|c|c|}
\hline \hline Algorithm & $(1)$ & $(2)$ & $(3)$ & $(4)$ & $(5)$ & $(6)$ & $(7)$ & $(8)$ & $(9)$ & $(10)$ \\
\hline NSGA-II-ToR (1) & - & $\circ$ & $\circ$ & $\circ$ & $\circ$ & $\circ$ & $\circ$ & $\circ$ & $\circ$ & $\circ$ \\
\hline ToP (2) & $\bullet$ & - & & $\circ$ & $\circ$ & $\circ$ & $\circ$ & $\circ$ & $\circ$ & $\circ$ \\
\hline CCMO (3) & $\bullet$ & & - & $\circ$ & & $\circ$ & $\circ$ & & $\circ$ & $\circ$ \\
\hline NSGA-III (4) & $\bullet$ & $\bullet$ & $\bullet$ & - & $\bullet$ & & $\circ$ & $\bullet$ & & $\circ$ \\
\hline I-DBEA (5) & $\bullet$ & $\bullet$ & & $\circ$ & - & $\circ$ & $\circ$ & $\circ$ & $\circ$ & $\circ$ \\
\hline PPS (6) & $\bullet$ & $\bullet$ & $\bullet$ & & $\bullet$ & - & $\circ$ & $\bullet$ & $\circ$ & $\circ$ \\
\hline C-TAEA (7) & $\bullet$ & $\bullet$ & $\bullet$ & $\bullet$ & $\bullet$ & $\bullet$ & - & $\bullet$ & $\bullet$ & $\circ$ \\
\hline TiGE-2 (8) & $\bullet$ & $\bullet$ & & $\circ$ & $\bullet$ & $\circ$ & $\circ$ & - & $\circ$ & $\circ$ \\
\hline DCNSGA-III (9) & $\bullet$ & $\bullet$ & $\bullet$ & & $\bullet$ & & $\circ$ & $\bullet$ & - & $\circ$ \\
\hline CMME (10) & $\bullet$ & $\bullet$ & $\bullet$ & $\bullet$ & $\bullet$ & $\bullet$ & $\bullet$ & $\bullet$ & $\bullet$ & - \\
\hline \hline
\end{tabular}

\section{B. Comparison With Other Algorithms}

In this section, CMME is compared with the aforementioned nine algorithms. The detailed HV and IGD results are respectively reported in Table S-I, Table S-III, and Table S-I, Table SIV in the supplementary file, where "NaN" indicates that the algorithm cannot find feasible solutions on this problem, and "null" means that the obtained feasible solution set is far away from the true PF. In addition, the multiple-problem analysis is also performed to further verify the performance of $\mathrm{CMME}^{1}$. The average rankings of all the algorithms by the Friedman test are shown in Table III. The summarized results by the Wilcoxon test on the HV and IGD metrics are given in Table IV and Table $\mathrm{V}$, respectively.

Next, the experimental results of CMME and algorithms in comparison in terms of HV and IGD on DTLZ, MW and DASCMOP are described. Then the deep analysis and summaries about these results are detailed.

1) Description About The Results: In terms of HV indicator on C-DTLZ and DC-DTLZ, the results in Table S-I, Table III, and Table IV indicate that:

- CMME obtained the best HV values on 14 cases, followed by PPS (11), C-TAEA (9), CCMO (6), NSGAIII (3), and DCNSGA-III (2). For NSGA-II-ToR, ToP, I-DBEA, and TiGE-2, there was no best HV value on any case.

- As shown in the last column of Table S-I, CMME significantly outperformed NSGA-II-ToR, ToP, CCMO, NSGAIII, I-DEBA, PPS, C-TAEA, TiGE-2, and DCNSGAIII on $38,37,26,22,38,29,19,36$, and 24 cases, respectively. However, CMME only lost on $2,4,8,4$, $3,10,8,3$, and 4 cases, respectively.

- CMME has the best ranking among the 10 algorithms as shown in Table III, followed by C-TAEA.

- Among all the compared CMaOEAs, CCMO performed well on most three-objective problems since it is tailored for CMOPs. PPS performed well on C1-DTLZ3 and DC-DTLZ3 instances, of which the PF and CPF are consistent and the ignorance of constraints at the push stage accelerates the convergence speed to the PF as well as the $\mathrm{CPF}$ in dealing with multi-model instances.

\footnotetext{
${ }^{1}$ To perform the multiple-problem analysis, "NaN" is set to be -10 on the $\mathrm{HV}$ metric; while "NaN" is 10 and "null" is 5 on IGD.
} 
- According to the multiple-problem analysis by the Wilcoxon test shown in Table IV, it is clear that, except for C-TAEA, CMME yielded significantly better results than the other algorithms on the whole.

With respect to IGD, from the results in Table S-II, Table III, and Table V, we can observe that:

- On 17 cases, CMME obtained the best IGD values, followed by CCMO (10), C-TAEA (9), NSGA-III (6), PPS (5), and TiGE-2 (4). We find that CCMO performed well on the three-objective functions; however, its performance deteriorated dramatically for the higher objective problems. CMME was more robust for the test CMaOPs at a variable number of objective functions.

- In addition, CMME obtained significantly better results on the majority of the test cases than NSGA-II-ToR (47), ToP (45), CCMO (30), NSGA-III (28), I-DEBA (47), PPS (35), C-TAEA (30), TiGE-2 (42), and DCNSGA-III (27).

- Again, CMME had the best IGD ranking among the 10 algorithms.

- Finally, CMME significantly outperformed the other nine algorithms based on the Wilcoxon test shown in Table V.

For DC3-DTLZ, the current $E_{\max }$ does not seem to be enough for most CMaOEAs to converge to the CPF; therefore, the results of $\mathrm{HV}$ and IGD contain many $\mathrm{NaN}$ and null values. Next, the final solution sets on DC3-DTLZ are plotted to further study the efficiency of CMME.

To get a more intuitive observation and for further comparisons, we select three-objective C1-DTLZ1, DC1-DTLZ3, DC2-DTLZ3 and DC3-DTLZ3; five-objective C2-DTLZ2 and DC2-DTLZ3; eight-objective DC1-DTLZ1; 10-objective DC1DTLZ3 and DC2-DTLZ1 and 15-objective DC3-DTLZ3 as representatives. The obtained feasible PFs of the 10 algorithms with the median $\mathrm{HV}$ value among 30 runs are plotted in Figures S-1 to S-10 in the supplementary file. From these figures, we can see that:

- On three-objective C1-DTLZ1, CMME, CCMO, and DCNSGA-III obtained the best PFs with the most promising convergence and diversity. The obtained PFs of other the seven algorithms are poor on either the convergence or diversity. On three-objective DC1-DTLZ3, only CMME finally converged to the CPF. On three-objective DC2DTLZ3 and DC3-DTLZ3, CMME was stuck in the outer layer.

- On five-objective C2-DTLZ2, NSGA-III yielded the best PF, CMME was slightly worse than NSGA-III on diversity.

- On eight-objective DC1-DTLZ1, C-TAEA achieved the best results while CMME and DCNSGA-III performed slightly worse on diversity. On 10-objective DC1-DTLZ3, although all the algorithms failed to converge to the CPF, CMME obtained the best convergence in dealing with multi-model and discontinuous feasible regions.

- On 10-objective DC2-DTLZ1, CMME performed the best on both convergence and diversity, while on fiveobjective DC3-DTLZ3, although all the CMaOEAs didn't converge to the CPF, CMME and C-TAEA obtained the best convergence and diversity.
- On 15-objective DC3-DTLZ3, CMME also obtained the best convergence.

In terms of DAS-CMOP and MW shown in Table S-III (HV) and Table S-IV (IGD), CMME outperformed the other algorithms on almost all of the six instances. As shown in Figure S-11, CMME finally approached the CPF expect for DAS-CMOP9. DAS-CMOP8 and MW8 are chosen for further presentation. The final solution sets obtained by CMME and CMaOEAs in comparison on DAS-CMOP8 and MW8 are presented in Figure S-12 and Figure S-13, respectively. As shown in these figures, CMME obtained better results than most of the other algorithms except for CCMO.

2) Analysis and Summaries About The Results: Generally, the types of CMaOPs on which CMME performs well, shows only competitiveness or performs poorly can be summarized as follows:

- CMME performs well on C1-DTLZ1, C2-DTLZ2, C3DTLZ4, DC1-DTLZ, MW4, DAS-CMOP7 and DASCMOP8.

- CMME is competitive with other algorithms on DC2DTLZ1, DC3-DTLZ, MW8 and MW14.

- CMME performs poor on C1-DTLZ3, DC2-DTLZ3 and DAS-CMOP9.

For C1-DTLZ1, C2-DTLZ2, C3-DTLZ4 with relatively simple constraints, the CDP is enough to handle their constraints. Additionally, $\theta$-dominance applied in CMME is efficient for dealing with regularly shaped CPFs. Therefore, CMME is suitable for these instances. For DC1-DTLZ1 and DC1-DTLZ3, the $\mathrm{R}^{\# 1}$ ranking increases algorithm's ability to explore discontinuous feasible regions as shown in Figure 4. Therefore, even when the CPFs were irregular, CMME obtained better results. For MW4, the PF is the same as the CPF, and there are no outer layer infeasible regions, so CDP is sufficient for handling constraints. The $\theta$-dominance is also suitable for its regular CPF shape, so CMME obtained a well distributed solution set. As for DASCMOP7 with convergence-hardness, $\mathrm{R}^{\# 2}$ ranking and the enhanced environmental selection strengthened the selection pressure so that CMME obtained better result. As for DASCMOP8 with feasibility-hardness, the CDP's prioritization of feasibility ensures the feasibility promotion. Together with the convergence ability provided by $\mathrm{R}^{\# 2}$ ranking, CMME also obtained a good result.

DC2-DTLZ and DC3-DTLZ have inner outer layer feasible regions, and with the help of $\mathrm{R}^{\# 1}$ ranking and the designed mating strategy, CMME could across the infeasible regions and reach the inner layers. However within the given times of function evaluations, none of these algorithms could deal with the multi-model DC2-DTLZ3 and DC3-DTLZ3 situations as shown in Figure S-3 to Figure S-4. As shown in Figure S-9 and Figure S-10, CMME reached closer to the CPF than other algorithms when $m$ increased. Therefore, the trend is obvious that CMME suites many-objective situations. For MW8 and MW14, CMME only obtained competitive results due to the irregular CPF feature.

For C1-DTLZ3 and DC2-DTLZ3, CMME was worse than PPS. For these multi-model instances, the designed push stage 
of PPS can efficiently push the population near the PF by ignoring constraints. Therefore although PPS could not obtain good diversity, it had better convergence. As for DAS-CMOP9, all these approaches could not obtain good results since DASCMOP9 has both feasibility and diversity-hardness. The poor performance on multi-model and diversity-hardness instances indicates the difficulty of CMME on diversity promotion.

According to the results of C-TAEA and CMME depicted in these figures, especially on three-objective instances, we can see that $\theta$-dominance applied in CMME significantly promotes diversity performance of the final results. Although the proposed enhanced environmental selection is based on the CA update framework of C-TAEA, the performance of CMME was much better than C-TAEA due to the help of two rankings ( i.e. $\mathrm{R}^{\# 1}$ and $\mathrm{R}^{\# 2}$ ) and the mating environmental selections.

Therefore, from our results and analysis, we conclude that by benefiting from the enhanced mating and environmental selections, CMME algorithm achieved a good balance of feasibility, convergence, and diversity on the CMaOPs with variable numbers of objective functions. It provided better or highly competitive results compared with nine state-of-theart algorithms. However, CMME lacks diversity promotion ability, which results in poor performance on multi-model and diversity-hardness instances.

\section{Influence of Different Components in CMME}

This section empirically discusses the influence of different components in CMME. There are four variants to be compared with the proposed CMME method:

- CMME-1: The traditional Pareto dominance replaces the $\theta$-dominance used in the environmental selection as shown in line 12 of Algorithm 4.

- CMME-2: The TRF substitutes for the $\mathrm{R}^{\# 1}$ in the mating selection to evaluate a solution.

- CMME-3: In the $\mathrm{R}^{\# 2}$ strategy, both the feasibility and nondomination are considered. In C-TAEA, only the feasibility is used. Therefore, in CMME-3, the nondomination is removed to verify the effectiveness of the $R^{\# 2}$ strategy.

- CMME-4: In the mating and environmental selections, the individual density and crowding distance are used for diversity promotion. In CMME-4, both of them are removed to show their influence on the performance of CMME.

Table S-V in the supplementary file reports the detailed HV and IGD results for the five CMME variants. The multipleproblem statistical results by the Friedman and Wilcoxon tests

TABLE VI

AVERAGE RANKINGS OF HV AND IGD BY THE FRIEDMAN TEST FOR DIFFERENT CMME VARIANTS

\begin{tabular}{|c|c|c|}
\hline \hline Algorithm & Ranking (HV) & Ranking (IGD) \\
\hline CMME-1 & 2.63 & 2.75 \\
\hline CMME-2 & 3.75 & 4.08 \\
\hline CMME-3 & 3.72 & 3.80 \\
\hline CMME-4 & $\mathbf{2 . 6 0}$ & $\mathbf{2 . 3 9}$ \\
\hline CMME & $\mathbf{2 . 3 0}$ & $\mathbf{1 . 9 8}$ \\
\hline \hline
\end{tabular}

TABLE VII

RESULTS OBTAINED BY THE WILCOXON TEST, WHERE CMME IS COMPARED WITH ITS VARIANTS.

\begin{tabular}{|c|c|c|c||c|c|c|}
\hline \hline \multirow{2}{*}{ CMME vs } & \multicolumn{3}{|c||}{ HV } & \multicolumn{3}{c|}{ IGD } \\
\cline { 2 - 7 } & $R^{+}$ & $R^{-}$ & $p$-value & $R^{+}$ & $R^{-}$ & $p$-value \\
\hline CMME-1 & 771.0 & 504.0 & $\geq 0.2$ & 881.0 & 394.0 & $\mathbf{1 . 4 7 E - 2}$ \\
\hline CMME-2 & 1116.0 & 159.0 & $\mathbf{7 . 2 4 E - 7}$ & 1224.5 & 50.5 & $\mathbf{5 . 9 7 E - 1 1}$ \\
\hline CMME-3 & 1141.0 & 134.0 & $\mathbf{1 . 3 1 E}-7$ & 1169.0 & 106.0 & $\mathbf{3 . 1 8 E}-9$ \\
\hline CMME-4 & 777.0 & 498.0 & $1.81 \mathrm{E}-1$ & 889.5 & 385.5 & $\mathbf{1 . 4 3 E - 2}$ \\
\hline \hline
\end{tabular}

are given in Tables VI and VII, respectively. From the results, it can be seen that:

- CMME vs CMME-1: CMME obtained significantly better results on 17 and 24 cases in terms of HV and IGD, respectively. CMME lost on nine and nine cases on $\mathrm{HV}$ and IGD. In the rest of the cases, both of them get similar results. The results in Table VII show that CMME obtained slightly better HV performance, however, it significantly outperformed CMME-1 on the IGD metric. This indicates that the strengthened selection pressure by the $\theta$-dominance in the environmental selection can improve the performance of CMME for CMaOPs.

- CMME vs CMME-2 and CMME vs CMME-3: CMME yielded significantly better results in the majority of the test cases compared with CMME-2 and CMME-3. It also consistently outperformed both CMME-2 and CMME-3 on $\mathrm{HV}$ and IGD according to the Wilcoxon test. The results mean that the proposed two ranking strategies play essential roles in CMME to effectively solve CMaOPs.

- $C M M E$ vs $C M M E-4$ : In the majority of the cases, both obtained similar results. CMME had slightly better HV results, but performed significantly better than CMME-4 on IGD. This phenomenon proves the efficiency of the individual density used in the mating selection and the crowding distance used in the environmental selection.

- The results in Table VI show that CMME consistently obtained the best average rankings on both HV and IGD. This means that the synergy of different components can improve on the performance of CMME for CMaOPs.

TABLE VIII

PARAMETER SETTINGS FOR THE 10 METHODS ON REAL-WORLD APPLICATION PROBLEMS.

\begin{tabular}{|l|c|c|c|r|}
\hline \hline Problem & $m$ & $n$ & $N$ & $E_{\max }$ \\
\hline Gear Box Design & 3 & 7 & 91 & 26,250 \\
\hline Multi-product Batch Plant & 3 & 10 & 91 & 26,250 \\
\hline Heat Exchanger Network Design & 3 & 9 & 91 & 26,250 \\
\hline
\end{tabular}

\section{Experiments on Real-World Problems}

In the last section of experiments, the efficacy of CMME was verified through experiments on real-world applications. Three instances were chosen from [49] as CMaOPs from the real-world applications: 1) Gear Box Design problem [55], 2) Multi-product Batch Plant problem [56], and 3) Heat Exchanger Network Design problem [57].

The parameter settings are shown in Table VIII, where $m$ and $n$ represent the number of objective functions and decision 
variables, respectively. $N, E_{\max }$ represent the population size and the maximum number of function evaluations. The results of HV are presented in Table S-VI in the supplementary file, from which we can see that generally CMME outperformed the other algorithms on these three instances except for NSGAIII.

For three-objective Gear Box Design, CMME is only worse than NSGA-III. For three-objective Multi-product Batch Plant and Heat Exchanger Network Design, CMME outperformed all the other algorithms.

To further analyze the results, we depict the final solution sets of CMME and the algorithms in comparison on Multiproduct Batch Plant (denoted as MpBP in the figures) in Figure S-14. From Figure S-14 we can see that CMME performed well on MpBP as shown in Figure S-14. The convergence and diversity performance of CMME were significantly better than the results of other methods.

In summary, CMME performed well on these threeobjective real-world application problems.

\section{CONCLUSions AND Future Work}

In this paper, we have proposed an enhanced CMaOEA with enhanced mating and environmental selections, referred to as CMME, for CMaOPs. Specifically, two ranking strategies were designed for evaluating the convergence and feasibility of individuals. In addition, an individual density estimation was developed to improve diversity. Also, the $\theta$-dominance has been embedded into the environmental selection to strengthen the selection pressure for better handling of the many-objective situations. We found that the synergy of different components in CMME achieves a good balance of feasibility, convergence, and diversity. The proposed CMME algorithm was extensively evaluated through 10 benchmark CMaOPs with a variable number of objective functions, six benchmark CMOPs with three objective functions and three real-world applications. CMME was compared with nine related algorithms. The results show that CMME is able to provide better or highly competitive performance on the whole.

However, there remains some limitations to be studied in the future. For example, the performance of CMME on the multimodel test instances (such as C-DTLZ3, DC-DTLZ3) needs to be further improved. Moreover, the diversity promotion ability of CMME needs to be improved. Additionally, combining the proposed two ranking strategies with other enhanced dominance relations may develop other improved CMaOEAs for handling $\mathrm{CMaOPs}$ effectively.

The Matlab source code of CMME can be obtained from the authors upon request.

\section{REFERENCES}

[1] J. Wang, Y. Zhou, Y. Wang, J. Zhang, C. L. P. Chen, and Z. Zheng, "Multiobjective vehicle routing problems with simultaneous delivery and pickup and time windows: Formulation, instances, and algorithms," IEEE Transactions on Cybernetics, vol. 46, no. 3, pp. 582-594, 2016.

[2] H. Farzin, M. Fotuhi-Firuzabad, and M. Moeini-Aghtaie, "A stochastic multi-objective framework for optimal scheduling of energy storage systems in microgrids," IEEE Transactions on Smart Grid, vol. 8, no. 1, pp. 117-127, 2017.
[3] B. Tan, H. Ma, Y. Mei, and M. Zhang, "Evolutionary multi-objective optimization for web service location allocation problem," IEEE Transactions on Services Computing, pp. 1-1, 2018.

[4] Z. Fan, W. Li, X. Cai, H. Li, C. Wei, Q. Zhang, K. Deb, and E. Goodman, "Difficulty adjustable and scalable constrained multiobjective test problem toolkit," Evolutionary Computation, vol. 28, no. 3, pp. 339-378, 2020.

[5] T. Lafeta, M. L. Bueno, C. Brasil, and G. Oliveira, "Meands: A manyobjective evolutionary algorithm based on non-dominated decomposed sets applied to multicast routing," Applied Soft Computing, vol. 62, 09 2017.

[6] Y. Xiang, Y. Zhou, Z. Zheng, and M. Li, "Configuring software product lines by combining many-objective optimization and SAT solvers," ACM Trans. Softw. Eng. Methodol., vol. 26, no. 4, 2018.

[7] D. Gong, J. Sun, and Z. Miao, "A set-based genetic algorithm for interval many-objective optimization problems," IEEE Trans. Evol. Comput., vol. 22, no. 1, pp. 47-60, 2018.

[8] J. Wang, T. Weng, and Q. Zhang, "A two-stage multiobjective evolutionary algorithm for multiobjective multidepot vehicle routing problem with time windows," IEEE Transactions on Cybernetics, vol. 49, no. 7, pp. 2467-2478, 2019.

[9] Y. Wang, Z. Cai, Y. Zhou, and W. Zeng, "An adaptive tradeoff model for constrained evolutionary optimization," IEEE Transactions on Evolutionary Computation, vol. 12, no. 1, pp. 80-92, 2008.

[10] K. Li, R. Chen, G. Fu, and X. Yao, "Two-archive evolutionary algorithm for constrained multiobjective optimization," IEEE Transactions on Evolutionary Computation, vol. 23, no. 2, pp. 303-315, 2019.

[11] B. Li, J. Li, T. Ke, and Y. Xin, "Many-objective evolutionary algorithms: A survey," ACM Computing Surveys, vol. 48, no. 1, pp. 1-35, 2015.

[12] Y. Liu, D. Gong, X. Sun, and Y. Zhang, "Many-objective evolutionary optimization based on reference points," Applied Soft Computing, vol. 50, 112016

[13] Y. Liu, D. Gong, J. Sun, and Y. Jin, "A many-objective evolutionary algorithm using A one-by-one selection strategy," IEEE Trans. Cybern., vol. 47, no. 9, pp. 2689-2702, 2017. [Online]. Available: https://doi.org/10.1109/TCYB.2016.2638902

[14] D. Gong, Y. Liu, and G. Yen, "A meta-objective approach for manyobjective evolutionary optimization," Evolutionary Computation, vol. 28 , pp. 1-25, 112018.

[15] B. Tessema and G. G. Yen, "A self adaptive penalty function based algorithm for constrained optimization," in 2006 IEEE International Conference on Evolutionary Computation, 2006, pp. 246-253.

[16] T. P. Runarsson and Xin Yao, "Stochastic ranking for constrained evolutionary optimization," IEEE Transactions on Evolutionary Computation, vol. 4, no. 3, pp. 284-294, 2000.

[17] Z. Ma and Y. Wang, "Evolutionary constrained multiobjective optimization: Test suite construction and performance comparisons," IEEE Transactions on Evolutionary Computation, vol. 23, no. 6, pp. 972-986, 2019.

[18] K. Deb, "Multi-objective optimization using evolutionary algorithms," England: John Wiley and Sons, Ltd, vol. 16, 012001.

[19] M. Garza-Fabre, G. T. Pulido, and C. A. C. Coello, "Ranking methods for many-objective optimization," in MICAI 2009: Advances in Artificial Intelligence, A. H. Aguirre, R. M. Borja, and C. A. R. Garciá, Eds. Berlin, Heidelberg: Springer Berlin Heidelberg, 2009, pp. 633-645.

[20] D. Saxena, T. Ray, K. Deb, and A. Tiwari, "Constrained many-objective optimization: A way forward," in 2009 IEEE Congress on Evolutionary Computation, CEC 2009, 2009, pp. 545 - 552.

[21] K. Deb, A. Pratap, S. Agarwal, and T. Meyarivan, "A fast and elitist multiobjective genetic algorithm: NSGA-II," IEEE Transactions on Evolutionary Computation, vol. 6, no. 2, pp. 182-197, 2002.

[22] Y. Yuan, H. Xu, B. Wang, and X. Yao, "A new dominance relationbased evolutionary algorithm for many-objective optimization," IEEE Transactions on Evolutionary Computation, vol. 20, no. 1, pp. 16-37, 2016.

[23] K. Deb, "An efficient constraint handling method for genetic algorithms," Computer Methods in Applied Mechanics and Engineering, vol. 186, no. 2 , pp. $311-338,2000$.

[24] H. Jain and K. Deb, "An evolutionary many-objective optimization algorithm using reference-point based nondominated sorting approach, part ii: Handling constraints and extending to an adaptive approach," IEEE Transactions on Evolutionary Computation, vol. 18, no. 4, pp. 602-622, 2014.

[25] Z. Ma, Y. Wang, and W. Song, "A new fitness function with two rankings for evolutionary constrained multiobjective optimization," IEEE Transactions on Systems, Man, and Cybernetics: Systems, pp. 1-12, 2019. 
[26] Y. Tian, T. Zhang, J. Xiao, X. Zhang, and Y. Jin, "A coevolutionary framework for constrained multi-objective optimization problems," IEEE Transactions on Evolutionary Computation, pp. 1-1, 2020.

[27] T. Takahama and S. Sakai, "Constrained optimization by the $\varepsilon$ constrained differential evolution with gradient-based mutation and feasible elites," in 2006 IEEE International Conference on Evolutionary Computation, 2006, pp. 1-8.

[28] R. Jiao, S. Zeng, C. Li, S. Yang, and Y. S. Ong, "Handling constrained many-objective optimization problems via problem transformation," IEEE Transactions on Cybernetics, pp. 1-14, 2020.

[29] Q. Zhu, Q. Zhang, and Q. Lin, "A constrained multiobjective evolutionary algorithm with detect-and-escape strategy," IEEE Transactions on Evolutionary Computation, vol. 24, no. 5, pp. 938-947, 2020.

[30] Z. Fan, W. Li, X. Cai, H. Huang, Y. Fang, Y. Yugen, J. Mo, C. Wei, and E. Goodman, "An improved epsilon constraint-handling method in moea/d for cmops with large infeasible regions," Soft Computing, vol. 23, 122019.

[31] Z. Fan, Z. Wang, W. Li, Y. Yuan, Y. You, Z. Yang, F. Sun, and J. Ruan, "Push and pull search embedded in an $\mathrm{m} 2 \mathrm{~m}$ framework for solving constrained multi-objective optimization problems," Swarm and Evolutionary Computation, vol. 54, p. 100651, 2020.

[32] C. Saha, S. Das, K. Pal, and S. Mukherjee, "A fuzzy rule-based penalty function approach for constrained evolutionary optimization," IEEE Transactions on Cybernetics, vol. 46, no. 12, pp. 2953-2965, 2016

[33] M. Ming, A. Trivedi, R. Wang, D. Srinivasan, and T. Zhang, "A dualpopulation based evolutionary algorithm for constrained multi-objective optimization," IEEE Transactions on Evolutionary Computation, pp. 1$1,2021$.

[34] Z. Liu and Y. Wang, "Handling constrained multiobjective optimization problems with constraints in both the decision and objective spaces," IEEE Transactions on Evolutionary Computation, vol. 23, no. 5, pp. 870-884, 2019.

[35] Y. Tian, Y. Zhang, Y. Su, X. Zhang, K. Tan, and Y. Jin, "Balancing objective optimization and constraint satisfaction in constrained evolutionary multi-objective optimization," IEEE Transactions on Cybernetics, 08 2020.

[36] Z. Fan, W. Li, X. Cai, H. Li, C. Wei, Q. Zhang, K. Deb, and E. Goodman, "Push and pull search for solving constrained multi-objective optimization problems," Swarm and Evolutionary Computation, vol. 44, pp. 665-679, 2019. [Online]. Available: https://www.sciencedirect.com/science/article/pii/S2210650218300233

[37] Q. Zhang and H. Li, "Moea/d: A multiobjective evolutionary algorithm based on decomposition," IEEE Transactions on Evolutionary Computation, vol. 11, no. 6, pp. 712-731, 2007.

[38] H. Liu, F. Gu, and Q. Zhang, "Decomposition of a multiobjective optimization problem into a number of simple multiobjective subproblems," IEEE Transactions on Evolutionary Computation, vol. 18, no. 3, pp. $450-455,2014$

[39] K. Deb and H. Jain, "An evolutionary many-objective optimization algorithm using reference-point-based nondominated sorting approach, part i: Solving problems with box constraints," IEEE Transactions on Evolutionary Computation, vol. 18, no. 4, pp. 577-601, 2014.

[40] M. Asafuddoula, T. Ray, and R. Sarker, "A decomposition-based evolutionary algorithm for many objective optimization," IEEE Transactions on Evolutionary Computation, vol. 19, no. 3, pp. 445-460, 2015.

[41] M. Elarbi, S. Bechikh, and L. B. Said, "On the importance of isolated solutions in constrained decomposition-based many-objective optimization," in Proceedings of the Genetic and Evolutionary Computation Conference, 2017, pp. 561-568.

[42] M. Elarbi, S. Bechikh, and L. Ben Said, "On the importance of isolated infeasible solutions in the many-objective constrained NSGAIII," Knowledge-Based Systems, p. 104335, 2018.

[43] M. Miyakawa, H. Sato, and Y. Sato, "Directed mating in decompositionbased moea for constrained many-objective optimization," in Proceedings of the Genetic and Evolutionary Computation Conference, 2018, pp. 721-728.

[44] J. Wang, G. Liang, and J. Zhang, "Cooperative differential evolution framework for constrained multiobjective optimization," IEEE Transactions on Cybernetics, vol. 49, no. 6, pp. 2060-2072, 2019.

[45] Y. Zhou, M. Zhu, J. Wang, Z. Zhang, Y. Xiang, and J. Zhang, "Trigoal evolution framework for constrained many-objective optimization," IEEE Transactions on Systems, Man, and Cybernetics: Systems, vol. 50, no. 8, pp. 3086-3099, 2020.

[46] C. Wang and R. Xu, "An angle based evolutionary algorithm with infeasibility information for constrained many-objective optimization," Applied Soft Computing, vol. 86, p. 105911, 2020.
[47] J. Cheng, G. G. Yen, and G. Zhang, "A many-objective evolutionary algorithm with enhanced mating and environmental selections," IEEE Transactions on Evolutionary Computation, vol. 19, no. 4, pp. 592-605, 2015.

[48] K. Li, K. Deb, Q. Zhang, and S. Kwong, "An evolutionary manyobjective optimization algorithm based on dominance and decomposition," IEEE Transactions on Evolutionary Computation, vol. 19, no. 5 , pp. 694-716, 2015.

[49] A. Kumar, G. Wu, M. Ali, R. Mallipeddi, P. Suganthan, and S. Das, "A test-suite of non-convex constrained optimization problems from the real-world and some baseline results," Swarm and Evolutionary Computation, vol. 56, p. 100693, 042020.

[50] Y. Tian, R. Cheng, X. Zhang, and Y. Jin, "PlatEMO: A MATLAB platform for evolutionary multi-objective optimization," IEEE Cотриtational Intelligence Magazine, vol. 12, pp. 73-87, 112017.

[51] R. Agrawal, K. Deb, and R. Agrawal, "Simulated binary crossover for continuous search space," Complex Systems, vol. 9, pp. 115-148, 06 2000.

[52] J. Alcalá-Fdez, L. Sánchez, S. García, M. J. del Jesus, S. Ventura, J. M. Garrell, J. Otero, C. Romero, J. Bacardit, V. M. Rivas, J. C. Fernández, and F. Herrera, "KEEL: A software tool to assess evolutionary algorithms for data mining problems," Soft Comput., vol. 13, no. 3, pp. 307-318, 2009.

[53] P. A. N. Bosman and D. Thierens, "The balance between proximity and diversity in multiobjective evolutionary algorithms," IEEE Transactions on Evolutionary Computation, vol. 7, no. 2, pp. 174-188, 2003.

[54] E. Zitzler and L. Thiele, "Multiobjective evolutionary algorithms: a comparative case study and the strength pareto approach," IEEE Transactions on Evolutionary Computation, vol. 3, no. 4, pp. 257-271, 1999.

[55] H.-Z. Huang, Y.-K. Gu, and X. Du, "An interactive fuzzy multi-objective optimization method for engineering design," Eng. Appl. of AI, vol. 19, pp. 451-460, 082006.

[56] G. Dhiman and V. Chahar, "Multi-objective spotted hyena optimizer: A multi-objective optimization algorithm for engineering problems," Knowledge-Based Systems, vol. 150, 032018.

[57] G. Guillén-Gosálbez, "A novel milp-based objective reduction method for multi-objective optimization: Application to environmental problems," Computers \& Chemical Engineering, vol. 35, pp. 1469-1477, 082011. 


\section{SUPPLEMENTARY FILES}

TABLE S-I

STATISTICAL RESULTS OF HV ON C-DTLZ AND DC-DTLZ OBTAINED BY CMME AND CMAOEAS IN COMPARISON, THE BEST VALUES OF EACH ROW ARE HIGHLIGHTED.

\begin{tabular}{|c|c|c|c|c|c|c|c|c|c|c|c|}
\hline $\begin{array}{ll}\text { Problem } \\
\end{array}$ & 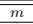 & $\begin{array}{l}\text { NSGA-II-ToR } \\
\end{array}$ & ToP & CCMO & NSSGA-III & I-DBEA & PPSS & "C-TAEA & T"TiGE-2 & "DCNSGA-III & CMME \\
\hline \multirow{5}{*}{ C1-DTLZ1 } & 3 & $\mathrm{NaN}(\mathrm{NaN})-$ & $\mathrm{NaN}(\mathrm{NaN})$ & $8.2301 \mathrm{e}-1(1.10 \mathrm{e}-2)$ & $8.2238 \mathrm{e}-1(1.28 \mathrm{e}-2)$ & $6.9752 \mathrm{e}-2(1.17 \mathrm{e}-1)$ & $7.2841 \mathrm{e}-1(1.28 \mathrm{e}$ & $8.2332 \mathrm{e}-1(1.34 \mathrm{e}-2)=$ & $2.7266 \mathrm{e}-1(1.36 \mathrm{e}$ & $8.2386 \mathrm{e}-1(1.10 \mathrm{e}-2)$ & $8 \mathrm{e}-1(8.3)$ \\
\hline & 5 & $(\mathrm{NaN})$ & $\mathrm{NaN}(\mathrm{NaN})$ & $9.4299 \mathrm{e}-1(2.18 \mathrm{e}-2)$ & $9.4182 \mathrm{e}-1(2.46 \mathrm{e}-2$ & $8.0668 \mathrm{e}-2(7.70 \mathrm{e}-2)$ & $6.5224 \mathrm{e}-1(2.9$ & 9.5910e-1 (1.18e-2) & $2.7411 \mathrm{e}-1(1.46 \mathrm{e}-1)$ & \begin{tabular}{|l|l|}
$9.4998 \mathrm{e}-1$ & $(1.86 \mathrm{e}-2$ \\
\end{tabular} & \\
\hline & ${ }^{\circ}$ & $1(1.64 \mathrm{e}-1)-$ & $\mathrm{N}$ & $9.4948 \mathrm{e}-1(4.3 \mathrm{C}$ & & $1.0011 \mathrm{e}-1(6.29)$ & & 07 & & & \\
\hline & 10 & e-1 $(2.50 \mathrm{e}-1)-$ & $\mathrm{NaN}(\mathrm{NaN})$ & $-2)-$ & $9.7546 \mathrm{e}-1(1$. & $-2(5.82 \mathrm{e}-2)$ & & 9.76 & & & \\
\hline & & -1) - & $\mathrm{NaN}(\mathrm{N}$ & & & & & 9.7946e-1 & & & \\
\hline \multirow{5}{*}{ C1-DTLZ3 } & & $0000 \mathrm{e}+0(0.00 \mathrm{e}+0)-$ & $2.4705 \mathrm{e}-1(2.18 \mathrm{e}-1)=$ & 6900e-1 (1.08e-1) + & $2.8905 \mathrm{e}-2(1.06 \mathrm{e}-1)$ & $6.3495 \mathrm{e}-4(3.48 \mathrm{e}-3)$ & $2.8598 \mathrm{e}-1(2.30 \mathrm{e}-1)=$ & $99 \mathrm{e}-3(2)$ & $+0(0)-4)-3$ & $9 \mathrm{e}-2(5$ & $142 \mathrm{e}-1$ \\
\hline & & & & & & & e-2 & & & & \\
\hline & & & & & & & & & & & \\
\hline & & $(0.00 \mathrm{e}+0)=$ & $0(0.00 e+0)=$ & $+0)=$ & $0.0000 e+0(0.00 e+0)=$ & $0.0000 \mathrm{e}+0$ & $4.8007 \mathrm{e}-2(8$ & $0.0000 \mathrm{e}+0(0.00 \mathrm{e}+0)=$ & $0.0000 \mathrm{e}+0(0.00 \mathrm{e}+0)=$ & $0.0000 \mathrm{e}+0$ & $0.0000 \mathrm{e}+0$ \\
\hline & 15 & $0.0000 \mathrm{e}+0(0.00 \mathrm{e}+0)=$ & $0.0000 \mathrm{e}+0(0.00 \mathrm{e}+0)=$ & $0.0000 \mathrm{e}+0(0.00 \mathrm{e}+0)=$ & $0.0000 e+0(0.00 e+0)=$ & $0.0000 \mathrm{e}+0(0.00 \mathrm{e}+0)=$ & $9.2683 \mathrm{e}-2(7.97 \mathrm{e}-2)+$ & $0.0000 \mathrm{e}+0(0.00 \mathrm{e}+0)=$ & $0.0000 \mathrm{e}+0(0.00 \mathrm{e}+0)=$ & $0.0000 \mathrm{e}+0(0.00 \mathrm{e}+0)=$ & $0.0000 \mathrm{e}+0(0.00 \mathrm{e}+0)$ \\
\hline \multirow{4}{*}{ C2-DTLZ2 } & 3 & $3.1813 \mathrm{e}-1(3.85 \mathrm{e}-2)-$ & & $5.1296 \mathrm{e}-1(1$ & & & $4.9116 \mathrm{e}-1(3.47 \mathrm{e}-3)-$ & & & & \\
\hline & & & & & & & 5.3 & & 6. & & \\
\hline & $\begin{array}{c}8 \\
10\end{array}$ & $\begin{array}{l}2.07199-1)(1 . \\
3.0716 \mathrm{e}-1(1 .\end{array}$ & $\begin{array}{l}2.54322 \mathrm{e}-1(9 \\
2.135 \mathrm{e}-1(7\end{array}$ & $\begin{array}{l}7.7402 \mathrm{e}-1(1.153 \mathrm{e}-2)- \\
8.1886 \mathrm{e}-1(9.81 \mathrm{e}-3)\end{array}$ & $\begin{array}{l}7.6828 \mathrm{e}-1(1.32 \mathrm{e}-1)= \\
7.7505 \mathrm{e}-1(1.66 \mathrm{e}-1)=\end{array}$ & $\begin{array}{l}4.2858 \mathrm{e}-2(5.46 \mathrm{e}-2)- \\
5.2371 \mathrm{e}-2(5.08 \mathrm{e}-2)\end{array}$ & $\begin{array}{l}5.2660 \mathrm{e}-1(3.30 \mathrm{e}-2)- \\
4.1622 \mathrm{e}-1(1.44 \mathrm{e}-1)-\end{array}$ & \begin{tabular}{|l|l|}
$7.93499 \mathrm{e}-1$ & 7 \\
$8.5991 \mathrm{e}-1$ & $(1$ \\
\end{tabular} & $\begin{array}{l}7.8145 \mathrm{e}-1)(1.1 \\
8.5433 \mathrm{e}-1(9.5\end{array}$ & \begin{tabular}{|l|}
$8.2046-1$ \\
$8438 \mathrm{e}-1(5$. \\
\end{tabular} & . $720 \mathrm{e}-2)$ \\
\hline & 15 & $1.5627 \mathrm{e}-1(2.90 \mathrm{e}-2)-$ & $2.1989 \mathrm{e}-1(9.51 \mathrm{e}-2)-$ & $8.8519 \mathrm{e}-1(1.86 \mathrm{e}-2)-$ & $8.1693 \mathrm{e}-1(5.34 \mathrm{e}-2)-$ & $3.2667 \mathrm{e}-2(3.86 \mathrm{e}-2)-$ & $2.4692 \mathrm{e}-1(1.26 \mathrm{e}-1)-$ & $9.5007 \mathrm{e}-1(3.12 \mathrm{e}-3)-$ & $9.2880 \mathrm{e}-1(7.49 \mathrm{e}-3)-$ & $8.1867 \mathrm{e}-1(7.73 \mathrm{e}-2)-$ & \\
\hline \multirow{5}{*}{ C3-DTLZ4 } & & $5.3318 \mathrm{e}-1(1.34 \mathrm{e}-1)-$ & $7.4972 \mathrm{e}-1(1.24 \mathrm{e}-2)-$ & $7.7839 \mathrm{e}-1(4.5$ & 7.83 & $6.0223 \mathrm{e}-1(9.56 \mathrm{e}-2)-$ & $7.3799 \mathrm{e}-1(3.8$ & & 7.179 & $-2)-$ & \\
\hline & & 7144 & 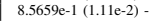 & & & & 8. & & 9 & & -2) \\
\hline & 8 & $1.8340 \mathrm{e}-1(6$ & $8.3952 \mathrm{e}-1(4$ & 6.68 & $9.8968 \mathrm{e}-1(1$ & $9.0628 \mathrm{e}-1(6.64 \mathrm{e}-2)$ & $9.5950 \mathrm{e}-1(8$. & & $9.7790 \mathrm{e}-1$ & $-2)-$ & 2-3) \\
\hline & & 1.6 & 8. & & 9.9 & 9.8 & 9.6 & & $9.8736 \mathrm{e}-1(4$ & $-2)$ - & e-4) \\
\hline & 15 & $1.1123 \mathrm{e}-1$ & $8.9944 \mathrm{e}-1(5$ & & 9.92 & e-3) - & $9.8545 \mathrm{e}-1(1$. & & 9.9 & & 6e-6) \\
\hline \multirow{4}{*}{ DCI-DTLZ1 } & \begin{tabular}{|l|l}
3 \\
5
\end{tabular} & $\begin{array}{l}0.0000 \\
0.000\end{array}$ & $\begin{array}{l}2.1523 \mathrm{e}-1(2) \\
54355-24\end{array}$ & $6.2508 \mathrm{e}-1$ & & & 4.8. & & & & \\
\hline & & & & & & & 1) - & & & & \\
\hline & 8 & $0.0000 \mathrm{e}+0(0.00 \mathrm{e}+0)-$ & $1.2265 \mathrm{e}-1(1.34 \mathrm{e}-1)-$ & $0.0000 \mathrm{e}+0(0$ & 7.922 & $4.1203 \mathrm{e}-1(2$. & $7.4470 \mathrm{e}-1 \quad(4.4$ & 7.8369e-1 (5.51e-3) - & $1.8561 \mathrm{e}-2$ & 7.84 & \\
\hline & $\begin{array}{l}10 \\
15\end{array}$ & $\begin{array}{l}0.0000 \mathrm{e} e+0 \\
0.0000+0\end{array}$ & $\begin{array}{l}7.3928 \mathrm{e}-2(1.1 \\
5.5698 \mathrm{e}-2(1.1\end{array}$ & $\mid \begin{array}{l}0.0000 \mathrm{e}+0 \\
0.0000 \mathrm{e}+0\end{array}$ & $\begin{array}{r}7.86 \\
7.56\end{array}$ & $\begin{array}{l}2.90 \\
2.88\end{array}$ & $\begin{array}{l}4 e^{-1}(2 \\
8-1(1\end{array}$ & $\begin{array}{l}7.7 \\
7.9\end{array}$ & 0.00 & 1)- - & e-4) \\
\hline \multirow{5}{*}{ DC1-DTLZ3 } & & 0.0000 & $\frac{5.5098 \mathrm{e}-2(1.1}{5.4870 \mathrm{e}-2(8.7}$ & \begin{tabular}{|l|}
$.0 .00079 \mathrm{e}-1$ \\
$.8679-1$ \\
\end{tabular} & 3.733 & \begin{tabular}{|l|l}
2.080 \\
0.000 \\
\end{tabular} & $\frac{0.4508 \mathrm{e}-1(1.40 \mathrm{e}-1)-}{1.8489 \mathrm{e}-1(2.03 \mathrm{e}-1)-}$ & \begin{tabular}{|l|l|}
1.94 \\
4.87
\end{tabular} & $\frac{2.0}{0.0}$ & \begin{tabular}{|l|l|}
3.0 \\
3.0
\end{tabular} & $\frac{(2-3)}{2 e-1)}$ \\
\hline & & & & & & & 1.3 & & & 3. & \\
\hline & 8 & & 4.16 & & & & 1.7 & & & & \\
\hline & & 0. & 0.4 & & & & 1.2 & & & & \\
\hline & 15 & 10)- & 1.995 & & & & & & & & \\
\hline \multirow{4}{*}{ DC2-DTLZ1 } & & & & & & & & & & & \\
\hline & & $\begin{array}{lll}\mathrm{NaN}(1) \\
\mathrm{NaN}(\mathrm{l})\end{array}$ & $\mathrm{NaN}(\mathrm{N}$ & 9.2885e-1 & $\begin{array}{l}7.61 \\
8.97\end{array}$ & 9.8 & 5.7 & & 3. & & $-1)$ \\
\hline & $\begin{array}{c}8 \\
10\end{array}$ & & $\begin{array}{lll}\mathrm{NaN} \\
\mathrm{NaN}\end{array}$ & 815 & & & $\begin{array}{l}9.4 \\
8.5\end{array}$ & & & & \\
\hline & 15 & & & & & & & & & & \\
\hline \multirow{5}{*}{ DC2-DTLZ3 } & & & & & & & 3. & & & & \\
\hline & 5 & & $\mathrm{~N}$ & $1.5686 \mathrm{e}-2$ & $1.4728 \mathrm{e}-2$ & & 3.1 & 6.1 & 3.4 & & $-2(5.62 \mathrm{e}-3)$ \\
\hline & 8 & & & & & 0.00 & 3.7 & 2.8 & & & \\
\hline & & & & & & & 1. & & & & \\
\hline & $\frac{15}{3}$ & & $\mathrm{~N}$ & & & & 2. & & & & \\
\hline \multirow{4}{*}{ DC3-DTLZ1 } & 5 & $\begin{array}{l}0.0000 \mathrm{e}+0(0.00 \mathrm{e}+0)- \\
0.000 \mathrm{e}+0(0.00 \mathrm{e}+0)\end{array}$ & $\begin{array}{l}9666-30-3(3.270-2)- \\
0000+0(0.00+0)-\end{array}$ & $\begin{array}{l}5.22296 \mathrm{e}-1(8.95 \mathrm{e}-3)+ \\
6.8272 \mathrm{e}-2(4.69-2)+\end{array}$ & $\begin{array}{l}5.0877 \mathrm{e}-2 \\
1.559 \mathrm{e}-2\end{array}$ & \begin{tabular}{|l|l|l|l}
$0.00000+c+c$ \\
$0.0000 e+c$
\end{tabular} & $\begin{array}{l}2.7410 \mathrm{e}-1(2.02 \mathrm{e}-1)= \\
8.0422 \mathrm{e}-4(2.75 \mathrm{e}-3)\end{array}$ & \begin{tabular}{|l|l|}
4.877 \\
8.656
\end{tabular} & $\begin{array}{l}0.000 \\
0.000\end{array}$ & \begin{tabular}{|l|l|}
$2.88488 \mathrm{e}-1$ \\
$5.580 \mathrm{e}-2$
\end{tabular} & $\begin{array}{l}2.5669 \mathrm{e}-1(2.28 \mathrm{e}-1) \\
2.4335 \mathrm{e}-2(4.14 \mathrm{e}-2)\end{array}$ \\
\hline & 8 & $0.0000 \mathrm{e}+0(0.00 \mathrm{e}+0)+$ & $0.0000 \mathrm{e}+0(0.00 \mathrm{e}+0)+$ & $\mathrm{NaN}(\mathrm{N}$ & $\mathrm{NaN}(\mathrm{NaN})$ & $0.0000 \mathrm{e}+0(0$ & $0.0000 \mathrm{e}+0$ & $\begin{array}{l}0.0000 \mathrm{e}+0 \\
0.000\end{array}$ & $0.0000 \mathrm{e}+0$ & $\mathrm{Na}$ & $\mathrm{NaN}$ \\
\hline & & & $0.0000 \mathrm{e}+0(0.00 \mathrm{e}+0)+$ & & & & & 0000 & & & \\
\hline & & & & & & & & & & & \\
\hline \multirow{4}{*}{ DC3-DTLZ3 } & & 0. & 0.0 & + & i) $=$ & & 7.22 & 1.4 & $=$ & $0)=$ & $0 \mathrm{e}+0)$ \\
\hline & 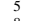 & & & & & & & & & & \\
\hline & 8 & & & & & & & & & & \\
\hline & $\begin{array}{l}10 \\
15\end{array}$ & $\begin{array}{l}0.00000+0+0(0.000 \mathrm{e}+0)= \\
0.000 \mathrm{e}+0(0.00 \mathrm{e}+0)=\end{array}$ & $\mid \begin{array}{l}0.0000 \mathrm{e}+0(0.00 \mathrm{e}+0)= \\
0.0000+0+0.00 \mathrm{e}+0)=\end{array}$ & $\mid \begin{array}{l}0.00000+0(0.00 e+0)= \\
0.0000+0+0(0.00+0)=\end{array}$ & $\begin{array}{l}0.00000+0+0(0.000+0)= \\
0.0000 \mathrm{e}+0(0.00 \mathrm{e}+0)=\end{array}$ & $\mid \begin{array}{l}0.0000+0+0(0.00 e+0)= \\
0.0000+0+0.00+0)=\end{array}$ & $\begin{array}{l}0.00000+0+0(0.000+0)= \\
0.0000 \mathrm{e}+0(0.00+0)=\end{array}$ & $\mid \begin{array}{l}0.0000+e+0(0.00 e+0)= \\
0.0000+0+0.00+0)=\end{array}$ & $\begin{array}{l}0.00000+0+0(0.00 \mathrm{e}+0)= \\
0.0000 \mathrm{e}+0.0 .00 \mathrm{e}+0)=\end{array}$ & $\begin{array}{l}0.0000 \mathrm{e}+0(\mathrm{C} \\
0.0000 \mathrm{e}+0\end{array}$ & \\
\hline & $2 / 38 / 10$ & $4 / 37 / 9$ & $8 / 26 / 16$ & $4 / 22 / 24$ & $3 / 38 / 9$ & $10 / 29 / 11$ & $8 / 19 / 23$ & $3 / 36 / 11$ & $4 / 24 / 22$ & \\
\hline
\end{tabular}

TABLE S-II

STATISTICAL RESULTS OF IGD OBTAINED By CMME AND CMAOEAS IN COMPARISON, THE BEST VALUES OF EACH ROW ARE HIGHLIGHTED.

\begin{tabular}{|c|c|c|c|c|c|c|c|c|c|c|c|}
\hline Problem & 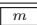 & A-II-ToR & $\overline{\mathrm{T} \text { ToP }}$ & CCMO & $\overline{\text { NSGA-1 }}$ & $\overline{\text { I-DBEA }}$ & $\overline{\overline{P P S}}$ & $\overline{C-T A E}$ & 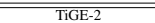 & $\overline{C \text { CNSGA-III }}$ & \\
\hline \multirow{5}{*}{ C1-DTLZI } & & & & $6 \mathrm{e}-2(4.0)$ & $33 \mathrm{e}-2(7.6$ & $73 \mathrm{e}-1(8.72$ & $\mathrm{e}-2(4$. & $57 \mathrm{e}-2(8$ & $31 \mathrm{e}-1(8$ & $7 \mathrm{e}-2(1.4 \mathrm{t}$ & $29 \mathrm{e}-2(8.37 \mathrm{e}-4)$ \\
\hline & 5 & $\mathrm{aN}(\mathrm{Na}$ & $\mathrm{NaN}(\mathrm{N}$ & $6.1770 \mathrm{e}-2(1.10 \mathrm{e}-3)=$ & $1098 \mathrm{e}-2(1.23)$ & $4.8638 \mathrm{e}-1(6.4$ & $1.9602 \mathrm{e}-1(1.23 \mathrm{e}-1)$ & $6.8812 \mathrm{e}-2(1.3$ & $3.8343 \mathrm{e}-1 \quad 7$. & $5.1297 \mathrm{e}-2(7.45$ & $1655 \mathrm{e}-2(1.43 \mathrm{e}-3)$ \\
\hline & 8 & $79 \mathrm{e}-1(8.62 \mathrm{e}-2)-$ & $\mathrm{NaN}(\mathrm{NaN})-$ & $1.1382 \mathrm{e}-1(1.66 \mathrm{e}-2)-$ & $9.5971 \mathrm{e}-2(1.80 \mathrm{e}-3)+$ & $4.9749 \mathrm{e}-1(4.14 \mathrm{e}-2)-$ & $1.3316 \mathrm{e}-1(1.84 \mathrm{e}-2)$ & $1.1656 \mathrm{e}-1(4.08 \mathrm{e}-3)$ & $4.4445 \mathrm{e}-1(5.24 \mathrm{e}-2)-$ & $1.0388 \mathrm{e}-1(1.83 \mathrm{e}-2)$ & $1.0076 \mathrm{e}-1(5.84 \mathrm{e}-3)$ \\
\hline & 10 & $3.1787 \mathrm{e}-1(1.25 \mathrm{e}-1)-$ & $\mathrm{NaN}(\mathrm{NaN})-$ & $1.2982 \mathrm{e}-1(1.66 \mathrm{e}-2)+$ & $1.3483 \mathrm{e}-1(4.80 \mathrm{e}-3)=$ & $5.4354 \mathrm{e}-1(4.90 \mathrm{e}-2)-$ & $2.0789 \mathrm{e}-1(3.88 \mathrm{e}-2)-$ & $1.3749 \mathrm{e}-1(4.84 \mathrm{e}-3)=$ & $4.0118 \mathrm{e}-1(6.91 \mathrm{e}-2)-$ & $1.3427 \mathrm{e}-1(1.21 \mathrm{e}-2)=$ & $1.3548 \mathrm{e}-1(4.0$ \\
\hline & 15 & $2.4414 \mathrm{e}-1(8.13 \mathrm{e}-2)-$ & $\mathrm{NaN}(\mathrm{NaN})-$ & $1.7295 \mathrm{e}-1(2.04 \mathrm{e}-2)-$ & $1.3448 \mathrm{e}-1(1.49 \mathrm{e}-2)=$ & $5.6635 \mathrm{e}-1(6.24 \mathrm{e}-2)-$ & $1.7975 \mathrm{e}-1(1.26 \mathrm{e}-2)-$ & $1.4721 \mathrm{e}-1(3.97 \mathrm{e}-3)-$ & $4.4785 \mathrm{e}-1(6.79 \mathrm{e}-2)-$ & $1.5618 \mathrm{e}-1(2.12 \mathrm{e}-2)-$ & $1.2922 \mathrm{e}-1(6.33 \mathrm{e}-3)$ \\
\hline \multirow{4}{*}{ C1-DTLZ3 } & & $1.5108 \mathrm{e}+1(2.88 \mathrm{e}+0)-$ & $.1470 \mathrm{e}+0(2.10 \mathrm{e}+0)=$ & $1.0071 \mathrm{e}-1(6.47 \mathrm{e}-2)+$ & $5.008 \mathrm{e}+0(3.57 \mathrm{e}+0)-$ & $7.5363 \mathrm{e}+0(1.90 \mathrm{e}+0)$ & $1.6700 \mathrm{e}+0(2.97 \mathrm{e}+0)=$ & $3.6163 \mathrm{e}+0(3.29 \mathrm{e}+0)$ & $7.0287 \mathrm{e}+0(2.73 \mathrm{e}+0)$ & $5.8904 \mathrm{e}+0(3.14 \mathrm{e}+0)-$ & $\frac{1.84}{2.842 \mathrm{e}+0(3.74 \mathrm{e}+0)}$ \\
\hline & 5 & $2.7682 \mathrm{e}+1(7$ & $085 \mathrm{e}+0$ & 1.142 & 1.13 & 1.1 & $e+0$ & $e+1($ & $+1(2.8$ & .0) + & -0) \\
\hline & 8 & $7.7613 \mathrm{e}+1(1.19 \mathrm{e}+1)-$ & $2.2427 \mathrm{e}+1$ & 1.19 & 1.05 & $1.0844 \mathrm{e}$ & $00 \mathrm{C} 2$ & 1.1 & $+1(4$ & 1.17 & +1 \\
\hline & 10 & $9.7744 \mathrm{e}+1(1.08 \mathrm{e}+1)-$ & $2.3411 \mathrm{e}+1(5.06 \mathrm{e}+0)-$ & $1.5414 \mathrm{e}+2(1$ & $5 e+1(1.2$ & 1. $4822 \mathrm{e}+1(2.68 \mathrm{e}-2)-$ & $5.7182 \mathrm{e}+0(6.10 \mathrm{e}+0)+$ & $1.3938 \mathrm{e}+1(1.5)$ & 1.1744e+1 $(5.49 \mathrm{e}+0)+$ & $\begin{array}{l}1.43 \\
151\end{array}$ & $4 e-2)$ \\
\hline \multirow{5}{*}{ C2-DTLZ2 } & $\frac{15}{3}$ & $\frac{1.1255+2+2(1.08 \mathrm{e}+1)-}{2.3394 \mathrm{e}-1(8.55 \mathrm{e}-2)}-$ & $\frac{2.7592 \mathrm{e}+1(6.26 \mathrm{e}+0)-}{9.1707 \mathrm{e}-2(1.62 \mathrm{e}-1)}$ & $\frac{1.8013 \mathrm{e}+2(6.75 \mathrm{e}+0)-}{4.5155 \mathrm{e}-2(5.06 \mathrm{e}-4)+}$ & $\frac{1.4679 \mathrm{e}+1(2.57 \mathrm{e}+0)-}{4.8218 \mathrm{e}-2(4.83 \mathrm{e}-4)+}$ & $\frac{1.4881 \mathrm{e}+1(3.94 \mathrm{e}-5)-}{8.284 \mathrm{le}-1(1.78 \mathrm{e}-1)-}$ & $\frac{1.28050+0(5.83 \mathrm{e}-1)+}{5.990-2-2.33 \mathrm{e}-3)}$ & $\begin{array}{l}1.4546 \mathrm{e}+1(6.04 \mathrm{e}-2)- \\
5.650 \mathrm{e}-2(1.44 \mathrm{e}-3)\end{array}$ & $\frac{1.3040 \mathrm{e}+1(4.41 \mathrm{e}+0)+}{1.8462 \mathrm{e}-1(3.63 \mathrm{e}-2)-}$ & $\frac{1.5136 \mathrm{e}+1(8.23 \mathrm{e}-1)-}{4.8188 \mathrm{e}-2(4.19 \mathrm{e}-4)+}$ & $\frac{1.4097 \mathrm{e}+1(1.97 \mathrm{e}+0)}{5.6008 \mathrm{e}-2(1.5 \mathrm{e}-3)}$ \\
\hline & & $4.7962 \mathrm{e}-1(1.84 \mathrm{e}-1)-$ & $2.4088 \mathrm{e}-1(1.99 \mathrm{e}-2)-$ & $1.6956 \mathrm{e}-1(3.01 \mathrm{e}-3)-$ & $2.0149 \mathrm{e}-1(1.14 \mathrm{e}-1)-$ & $1.0887 \mathrm{e}+0(1$. & $2.8552 \mathrm{e}-1(4.4$ & $1.7228 \mathrm{e}-1(1.7$ & $2.7104 \mathrm{e}-1(1.94 \mathrm{e}-2)-$ & $\begin{array}{l}4.01 \\
1.68 \\
\end{array}$ & $1.6585 \mathrm{e}-1(2.54 \mathrm{e}-3)$ \\
\hline & 8 & $8.6423 \mathrm{e}-1(1$ & $7.4277 \mathrm{e}-1(2$ & 2.79 & $3.4272 \mathrm{e}-1)(1$ & 1.2 & $4.9568 \mathrm{e}-1$ & 2.37 & 2.97 & 2) - & $\begin{array}{l}1.0300 \\
2.378\end{array}$ \\
\hline & & 7.701 & $8.5290 \mathrm{e}-1(1$ & & $5.2545 \mathrm{e}-1(1$ & $1.269 \mathrm{le}+0$ & $6.6648 \mathrm{e}-1(1.31 \mathrm{e}-1)-$ & 3.54 & $4.2104 \mathrm{e}-1(3$ & 4.66 & $3.3306 \mathrm{e}-1$ \\
\hline & 15 & $1.0562 \mathrm{e}+0(2.99 \mathrm{e}-2)-$ & $1.0019 \mathrm{e}+0(8.82 \mathrm{e}-2)-$ & $2.9789 \mathrm{e}-1(2.87 \mathrm{e}-2)-$ & $5.2008 \mathrm{e}-1(1.0$ & $1.3814 \mathrm{e}+0(8.87 \mathrm{e}-2)$ & $8.6529 \mathrm{e}-1(8.73 \mathrm{e}-2)-$ & $2.3290 \mathrm{e}-1(2.25 \mathrm{e}-2)+$ & $3.2893 \mathrm{e}-1(4.08 \mathrm{e}-3)-$ & 5.06 & $2.6385 \mathrm{e}-1$ \\
\hline \multirow{4}{*}{ C3-DTLZ4 } & 3 & $6.1403 \mathrm{e}-1(3.59 \mathrm{e}-1)-$ & $1.5711 \mathrm{e}-1(4.8$ & $1.2585 \mathrm{e}-1(1.35 \mathrm{e}-1)+$ & & $4.6315 \mathrm{e}-1(2.83 \mathrm{e}-1)-$ & $2.5327 \mathrm{e}-1(1.24 \mathrm{e}-1)-$ & & $2.5907 \mathrm{e}-1(2.40 \mathrm{e}-2)-$ & $1.9740 \mathrm{e}-1(2.22 \mathrm{e}-1)=$ & $1.3273 \mathrm{e}-1(1.34 \mathrm{e}-1)$ \\
\hline & & 7.477 & 5.210 & 3.57 & & 7. & 5.5 & & & & \\
\hline & 8 & 1.634 & $9.4007 \mathrm{e}-1(6$. & 2.090 & & 8.967 & $7.6735 \mathrm{e}-1)(3$ & 5.42 & 7.00 & 7.5 & $-2)$ \\
\hline & \begin{tabular}{|l}
10 \\
15
\end{tabular} & $\begin{array}{l}1.8318 \mathrm{e}+0(7.21 \mathrm{e}-2) \\
2.1172 \mathrm{e}+0 \\
2.32 \mathrm{e}-2)\end{array}$ & $\begin{array}{l}1.0862 \mathrm{e}+0(6.09 \mathrm{e}-2)- \\
1.2736 \mathrm{e}+0(671 \mathrm{e}-2)\end{array}$ & $\begin{array}{l}2.2554 \mathrm{e}+0(1.70 \mathrm{e}-2)- \\
2.4323 \mathrm{e}+0(112 \mathrm{e}-2)=\end{array}$ & $\begin{array}{l}6.7171 \mathrm{e}-1(1.35 \mathrm{e}-1)= \\
8.9523 \mathrm{e}-1(2.19 \mathrm{e}-1)=\end{array}$ & $\begin{array}{l}8.4632 \mathrm{e}-1(7.98 \mathrm{e}-2)- \\
96540 \mathrm{e}-1(5.37 \mathrm{e}-2)\end{array}$ & $\begin{array}{l}9.0021 \mathrm{e}-1(8.07 \mathrm{e}-2)- \\
10239 \mathrm{e}+0(576 \mathrm{e}-2)\end{array}$ & $\begin{array}{l}6.2920 \mathrm{e}-1(2.76 \mathrm{e}-3)- \\
7.3228 \mathrm{e}-1(3.46 \mathrm{e}-3)\end{array}$ & $\begin{array}{l}8.4094 \mathrm{e}-1(3.09 \mathrm{e}-2) \\
9.6418 \mathrm{e}-1(2.84 \mathrm{e}-2)-\end{array}$ & $\begin{array}{l}9.3604 \mathrm{e}-1(9.04 \mathrm{e}-2)- \\
1.1512 \mathrm{e}+0 \\
1.23 \mathrm{e}-1)\end{array}$ & $\begin{array}{l}6.0301 \mathrm{e}-1(1.51 \mathrm{e}-2) \\
7.1515 \mathrm{e}-1 \\
(8.11 \mathrm{e}-3)\end{array}$ \\
\hline \multirow{5}{*}{ DCl-DTLZ1 } & & $1.7954 \mathrm{e}+1(5.67 \mathrm{e}+0)-$ & $5.2685 \mathrm{e}-1(6.5)$ & $1.2906 \mathrm{e}-2(7$. & $\frac{0.972}{1.441}$ & $2.698 \mathrm{le}-1(2.26 \mathrm{e}-1)$ & $8.2207 \mathrm{e}-2(1.50 \mathrm{e}-1)-$ & $1.8300 \mathrm{e}-2(1.42 \mathrm{e}-2)-$ & $1.9810 \mathrm{e}+0(1.15 \mathrm{e}+0)-$ & $(e-4)+$ & $1.5811 \mathrm{e}-2$ \\
\hline & & 1.54 & 1.57 & & 4.74 & 2.88 & 3. & & & & \\
\hline & 8 & $6.4789 \mathrm{e}+1(1.4$ & 2.517 & 2.76 & $8.4112 \mathrm{e}-2(8)$ & $2.731 \mathrm{le}-1(2$ & 1.1 & 8.5 & 1.3 & 9.0 & -3) \\
\hline & & & & & & & 2.9 & & & & \\
\hline & 15 & 8.583 & 4.262 & & 1.46 & 3.331 & $2.0174 \mathrm{e}-1$ & 1.28 & & & \\
\hline \multirow{5}{*}{ DCl-DTLZ3 } & 3 & 1.5625 & 2.617 & & 1.77 & & 1.0 & 4.59 & & & \\
\hline & 5 & & & & & & & & & & \\
\hline & 8 & $7.1608 \mathrm{e}+1(1.04 \mathrm{e}+1)-$ & $1.7867 \mathrm{e}+1(8.2$ & 9.83 & 7.283 & 2.101 & 1.49 & 1.2 & & & \\
\hline & 10 & 8.5230 & 2.349 & & & & 5.2. & & & & \\
\hline & 15 & $1.1011 \mathrm{e}+2(9$ & $2.3745 \mathrm{e}+1(9)$ & $\frac{1.75}{2.07}$ & $5.9842 \mathrm{e}+0(2.44 \mathrm{e}+0)-$ & $1.6068 \mathrm{e}+0$ & $1.4457 \mathrm{e}+0(1.0$ & & & & \\
\hline \multirow{4}{*}{ DC2-DTLZ1 } & 5 & & $\begin{array}{l}\mathrm{NaN}(\mathrm{Na} \\
\mathrm{NaN}\end{array}$ & $\begin{array}{l}2.1975 \mathrm{e}-2(9) \\
8.9018-2(3)\end{array}$ & $\begin{array}{l}1.4919 \mathrm{e}-1(6.03 \mathrm{e}-2)- \\
1.6464 \mathrm{e}-1(5.14 \mathrm{e}-2)-\end{array}$ & $\begin{array}{l}4.1004 \mathrm{e}-1(1.18 \mathrm{e}-1)- \\
4.2894 \mathrm{e}-1\end{array}$ & $\begin{array}{l}4.0238 \mathrm{e}-2(3.02 \mathrm{e}-2)+ \\
2.2296-1\end{array}$ & $\begin{array}{l}3.1135 \mathrm{e}-2(2.7 \\
8.3915 \mathrm{e}-2(3 .\end{array}$ & $5 e-1(8$ & 2.3 & $4.9399 \mathrm{e}-2(6.71 \mathrm{e}-2)$ \\
\hline & 8 & & $\mathrm{NaN}$ & POI & & & $\begin{array}{l}2.2 \\
1.5\end{array}$ & 8. & 3.4 & 1. & $1.1547 \mathrm{e}-1(5.57 \mathrm{e}-2)$ \\
\hline & & & & 4.99 & & & & & & & \\
\hline & 15 & & & & & & & & & & \\
\hline \multirow{5}{*}{ DC2-DTLZ3 } & 3 & & & 4.05 & & & 3. & & & & -2) \\
\hline & & & & & 8.384 & & & & & & \\
\hline & 8 & $\mathrm{NaN}(\mathrm{I})$ & & & $\mathrm{N}$ & 1.583 & 7.8 & & & & $-1)$ \\
\hline & 10 & $\mathrm{NaN}$ & & & 1.018 & & & & & & \\
\hline & & $\frac{N i_{i}}{7.3786}$ & $\frac{\mathrm{NaN}(\mathrm{Na}}{677 \mathrm{e}+0)}$ & 8.928 & & $\frac{1.74}{1.69}$ & $\frac{1.05}{4.35}$ & $\frac{9.20290 \mathrm{e}-1(1.63 \mathrm{e}-2)=}{1.8860 \mathrm{e}-2(1.06 \mathrm{e}) 2)}$ & $\frac{-1)-}{+0)-}$ & $\frac{e+0(3.98 \mathrm{e}-2)-}{\mathrm{e}-1(1.97 \mathrm{e}-1)=}$ & $1.3314 \mathrm{e}-1$ \\
\hline \multirow{4}{*}{ DC3-DTLZ1 } & 5 & $7.3947 \mathrm{e}+1(2.67 \mathrm{e}+1)$ & $1.9390 \mathrm{e}+1(8.45 \mathrm{e}+0)-$ & $8.3865 \mathrm{e}-2(1.00 \mathrm{e}-1)+$ & $2.7484 \mathrm{e}-1(2.48 \mathrm{e}-1)=$ & $3.3981 \mathrm{e}+1$ & $7.9032 \mathrm{e}+0$ & $5.4425 \mathrm{e}-1$ & $3.8926 \mathrm{et}-\mathrm{C}$ & $1.2649 \mathrm{e}-1$ & $22 \mathrm{e}-1)$ \\
\hline & 8 & nul & null & $\mathrm{Na}$ & $\mathrm{Na}$ & $\mathrm{nul}$ & in & & & & \\
\hline & & & & & & & & & & & \\
\hline & & $\mathrm{nu}$ & $\mathrm{nu}$ & & & & & & & & \\
\hline \multirow{4}{*}{ DC3-DTLZ3 } & 3 & 6.1070 & 1.2754 & 5.7354e-1 & & 1.122 & & & & & \\
\hline & & & & & & & & & & & \\
\hline & $\begin{array}{c}8 \\
10\end{array}$ & $\begin{array}{l}5.9374 \\
59214\end{array}$ & & & & & & & $679>2$ & 755 & $-1)$ \\
\hline & 10 & $5.9214 \mathrm{e}$ & 625 & 8.288 & $\begin{array}{l}\text {.1.1800e+0 } \\
1.0002 \mathrm{e}+1\end{array}$ & $\begin{array}{l}9.1064 \mathrm{e}+0 \\
1.0201 \mathrm{e}+1\end{array}$ & $\begin{array}{l}2.43499+18 \\
2.3272 e+1(4\end{array}$ & $\begin{array}{l}4.708 \mathrm{e}+0(1) \\
3.636 \mathrm{le}+0(1\end{array}$ & $6.6377 \mathrm{e}+0(1)$ & $\begin{array}{l}7.5884+2+0 \\
1.4022+1\end{array}$ & $1.5789 \mathrm{et}-\mathrm{C}$ \\
\hline & & $2 / 4$ & $4 / 45$ & $1 / 30 / 9$ & $8 / 28 / 14$ & $3 / 47 / 0$ & $7 / 35 / 8$ & $8 / 30 / 12$ & $7 / 42 / 1$ & $5 / 27 / 18$ & \\
\hline
\end{tabular}


TABLE S-III

STATISTICAL RESULTS OF HV ON DAS-CMOP AND MW OBTAINED BY CMME AND CMAOEAS IN COMPARISON, THE BEST VALUES OF EACH ROW ARE HIGHLIGHTED.

\begin{tabular}{|c|c|c|c|c|c|c|c|c|c|c|c|}
\hline Problem & $m$ & NSGA-II-ToR & ToP & ССMO & NSGA-III & I-DBEA & PPS & C-TAEA & TiGE-2 & DCNSGA-III & CMME \\
\hline DASCMOP7 & 3 & $\mathrm{NaN}(\mathrm{NaN})$ & $\mathrm{NaN}(\mathrm{NaN})$ & $2.7075 \mathrm{e}-1(3.04 \mathrm{e}-2)-$ & $2.2237 \mathrm{e}-1(4.04 \mathrm{e}-2)-$ & $5.6234 \mathrm{e}-3(1.01 \mathrm{e}-2)-$ & $1.2177 \mathrm{e}-1(7.92 \mathrm{e}-2)-$ & $2.1566 \mathrm{e}-1(6.34 \mathrm{e}-2)-$ & $1.2804 \mathrm{e}-2(2.01 \mathrm{e}-2)-$ & $2.2156 \mathrm{e}-1(6.19 \mathrm{e}-2)-$ & $2.7945 \mathrm{e}-1(2.63 \mathrm{e}-2)$ \\
\hline DASCMOP8 & 3 & $\mathrm{NaN}(\mathrm{NaN})$ & $\mathrm{NaN}(\mathrm{NaN})$ & $1.8360 \mathrm{e}-1(3.04 \mathrm{e}-2)-$ & $1.4028 \mathrm{e}-1(3.04 \mathrm{e}-2)-$ & $1.1779 \mathrm{e}-3(6.34 \mathrm{e}-3)-$ & $5.6646 \mathrm{e}-2(6.52 \mathrm{e}-2)-$ & $1.1887 \mathrm{e}-1(5.20 \mathrm{e}-2)-$ & $1.4113 \mathrm{e}-2(2.79 \mathrm{e}-2)-$ & $1.4179 \mathrm{e}-1(6.28 \mathrm{e}-2)-$ & $1.9807 \mathrm{e}-1(1.48 \mathrm{e}-2)$ \\
\hline DASCMOP9 & 3 & $5.5079 \mathrm{e}-2(8.02 \mathrm{e}-3)-$ & $1.4048 \mathrm{e}-2(1.57 \mathrm{e}-2)-$ & $1.1724 \mathrm{e}-1(1.43 \mathrm{e}-2) \approx$ & $9.4793 \mathrm{e}-2(1.81 \mathrm{e}-2)-$ & $4.1708 \mathrm{e}-2(9.71 \mathrm{e}-3)-$ & $7.8483 \mathrm{e}-2(4.31 \mathrm{e}-2)-$ & $1.3353 \mathrm{e}-1(1.14 \mathrm{e}-2)+$ & $8.0835 \mathrm{e}-2(1.77 \mathrm{e}-2)-$ & $1.0658 \mathrm{e}-1(1.49 \mathrm{e}-2)-$ & $1.1594 \mathrm{e}-1(1.68 \mathrm{e}-2)$ \\
\hline MW4 & 3 & $2.3305 \mathrm{e}-1(1.04 \mathrm{e}-1)-$ & $\mathrm{NaN}(\mathrm{NaN})$ & $8.3689 \mathrm{e}-1(1.22 \mathrm{e}-3)-$ & $8.2297 \mathrm{e}-1(6.36 \mathrm{e}-2)-$ & $2.1507 \mathrm{e}-1(9.92 \mathrm{e}-2)-$ & $6.7280 \mathrm{e}-1(0.00 \mathrm{e}+0) \approx$ & $8.3565 \mathrm{e}-1(9.59 \mathrm{e}-4)-$ & $7.9304 \mathrm{e}-1(1.10 \mathrm{e}-2)-$ & $8.3712 \mathrm{e}-1(1.32 \mathrm{e}-3)-$ & $8.4134 \mathrm{e}-1(2.26 \mathrm{e}-4)$ \\
\hline MW8 & 3 & $2.6511 \mathrm{e}-1(8.19 \mathrm{e}-2)-$ & $4.2726 \mathrm{e}-2(6.85 \mathrm{e}-2)-$ & $5.2588 \mathrm{e}-1(1.17 \mathrm{e}-2)+$ & $5.0414 \mathrm{e}-1(5.73 \mathrm{e}-2) \approx$ & $5.0069 \mathrm{e}-2(3.27 \mathrm{e}-2)-$ & $3.2387 \mathrm{e}-1(7.29 \mathrm{e}-2)-$ & $5.2027 \mathrm{e}-1(1.41 \mathrm{e}-2) \approx$ & $4.4676 \mathrm{e}-1(4.65 \mathrm{e}-2)-$ & $5.2739 \mathrm{e}-1(1.36 \mathrm{e}-2)+$ & $5.1920 \mathrm{e}-1(1.21 \mathrm{e}-2)$ \\
\hline MW14 & 3 & $3.7720 \mathrm{e}-2(5.28 \mathrm{e}-3)-$ & $2.4309 \mathrm{e}-1(1.64 \mathrm{e}-1)-$ & $4.6360 \mathrm{e}-1(1.46 \mathrm{e}-2)-$ & $3.1773 \mathrm{e}-1(9.73 \mathrm{e}-2)-$ & $1.1050 \mathrm{e}-2(2.49 \mathrm{e}-3)-$ & $3.4221 \mathrm{e}-1(5.12 \mathrm{e}-2)-$ & $4.4328 \mathrm{e}-1(4.49 \mathrm{e}-2)-$ & $4.3593 \mathrm{e}-1(8.03 \mathrm{e}-3)-$ & $4.4775 \mathrm{e}-1(2.85 \mathrm{e}-2)-$ & $4.6499 \mathrm{e}-1(2.69 \mathrm{e}-3)$ \\
\hline$+1-1 \approx$ & & $0 / 4 / 0$ & $0 / 3 / 0$ & $1 / 4 / 1$ & $0 / 5 / 1$ & $0 / 6 / 0$ & $0 / 5 / 1$ & $1 / 4 / 1$ & $0 / 6 / 0$ & $1 / 5 / 0$ & \\
\hline
\end{tabular}

TABLE S-IV

STATISTICAL RESULTS OF IGD ON DAS-CMOP AND MW OBTAINED BY CMME AND CMAOEAS IN COMPARISON, THE BEST VALUES OF EACH ROW ARE HIGHLIGHTED.

\begin{tabular}{|c|c|c|c|c|c|c|c|c|c|c|c|}
\hline Problem & $m$ & NSGA-II-ToR & ToP & СCMO & NSGA-III & I-DBEA & PPS & C-TAEA & TiGE-2 & DCNSGA-III & CMME \\
\hline DASCMOP7 & 3 & $\mathrm{NaN}(\mathrm{NaN})$ & $\mathrm{NaN}(\mathrm{NaN})$ & $6.2130 \mathrm{e}-2(6.29 \mathrm{e}-2) \approx$ & $1.8095 \mathrm{e}-1(1.03 \mathrm{e}-1)-$ & $1.1243 e+0(2.38 \mathrm{e}-1)-$ & $4.8136 \mathrm{e}-1(3.22 \mathrm{e}-1)-$ & $1.7651 \mathrm{e}-1(1.54 \mathrm{e}-1)-$ & $9.3925 \mathrm{e}-1(2.68 \mathrm{e}-1)-$ & $1.6438 \mathrm{e}-1(1.32 \mathrm{e}-1)-$ & $5.2590 \mathrm{e}-2(5.13 \mathrm{e}-2)$ \\
\hline DASCMOP8 & 3 & $\mathrm{NaN}(\mathrm{NaN})$ & $\mathrm{NaN}(\mathrm{NaN})$ & $9.8580 \mathrm{e}-2(8.76 \mathrm{e}-2) \approx$ & $2.3978 \mathrm{e}-1(1.27 \mathrm{e}-1)-$ & $1.3219 \mathrm{e}+0(1.29 \mathrm{e}-1)-$ & $5.9561 \mathrm{e}-1(3.91 \mathrm{e}-1)-$ & $2.3498 \mathrm{e}-1(1.46 \mathrm{e}-1)-$ & $7.2264 \mathrm{e}-1(2.56 \mathrm{e}-1)-$ & $1.6858 \mathrm{e}-1(1.16 \mathrm{e}-1)-$ & $7.3034 \mathrm{e}-2(.93 \mathrm{e}-2)$ \\
\hline DASCMOP9 & 3 & $6.5264 \mathrm{e}-1(9.78 \mathrm{e}-2)-$ & $8.5863 \mathrm{e}-1(9.52 \mathrm{e}-2)-$ & $3.9291 \mathrm{e}-1(6.79 \mathrm{e}-2) \approx$ & $4.8820 \mathrm{e}-1(9.43 \mathrm{e}-2)-$ & $7.5553 \mathrm{e}-1(8.83 \mathrm{e}-2)-$ & $5.0891 \mathrm{e}-1(1.73 \mathrm{e}-1)-$ & $2.8553 \mathrm{e}-1(5.96 \mathrm{e}-2)+$ & $4.2496 \mathrm{e}-1(6.44 \mathrm{e}-2) \approx$ & $4.3325 \mathrm{e}-1(7.82 \mathrm{e}-2) \approx$ & $4.2342 \mathrm{e}-1$ \\
\hline MW4 & 3 & $5.4095 \mathrm{e}-1(1.68 \mathrm{e}-1)-$ & $\mathrm{NaN}(\mathrm{NaN})$ & $4.3957 \mathrm{e}-2(6.14 \mathrm{e}-4)-$ & $5.4631 \mathrm{e}-2(5.2 \mathrm{le}-2)-$ & $5.6394 \mathrm{e}-1(1.53 \mathrm{e}-1)-$ & $1.0982 \mathrm{e}-1(0.00 \mathrm{e}+0) \approx$ & $4.8368 \mathrm{e}-2(8.89 \mathrm{e}-4)-$ & $8.4043 \mathrm{e}-2(7.11 \mathrm{e}-3)-$ & $4.5136 \mathrm{e}-2(1.29 \mathrm{e}-3)-$ & $4.1393 \mathrm{e}-2(2.76 \mathrm{e}-4)$ \\
\hline MW8 & 3 & $3.4824 \mathrm{e}-1(1.81 \mathrm{e}-1)-$ & $9.2960 \mathrm{e}-1(1.93 \mathrm{e}-1)-$ & $4.9510 \mathrm{e}-2(3.54 \mathrm{e}-3)+$ & $7.0324 \mathrm{e}-2(7.59 \mathrm{e}-2) \approx$ & $7.8075 \mathrm{e}-1(1.44 \mathrm{e}-1)-$ & $1.4833 \mathrm{e}-1(4.37 \mathrm{e}-2)-$ & $5.4420 \mathrm{e}-2(3.60 \mathrm{e}-3)+$ & $1.2600 \mathrm{e}-1(2.68 \mathrm{e}-2)-$ & $5.0326 \mathrm{e}-2(3.34 \mathrm{e}-3)+$ & $5.6446 \mathrm{e}-2(3.92 \mathrm{e}-3)$ \\
\hline MW14 & 3 & $1.3966 \mathrm{e}+0(1.25 \mathrm{e}-1)-$ & $9.9489 \mathrm{e}-1(9.20 \mathrm{e}-1)-$ & $1.1784 \mathrm{e}-1(3.59 \mathrm{e}-2)+$ & $4.0845 \mathrm{e}-1(1.92 \mathrm{e}-1)-$ & $2.6350 \mathrm{e}+0(5.51 \mathrm{e}-1)-$ & $4.6101 \mathrm{e}-1(1.96 \mathrm{e}-1)-$ & $1.5382 \mathrm{e}-1(1.08 \mathrm{e}-1)-$ & $1.9458 \mathrm{e}-1(2.14 \mathrm{e}-2)-$ & $1.6734 \mathrm{e}-1(7.11 \mathrm{e}-2)-$ & $1.3421 \mathrm{e}-1(5.94 \mathrm{e}-3)$ \\
\hline$+/-/ \approx$ & & $0 / 4 / 0$ & $0 / 3 / 0$ & $2 / 1 / 3$ & $0 / 5 / 1$ & $0 / 6 / 0$ & $0 / 5 / 1$ & $2 / 4 / 0$ & $0 / 5 / 1$ & & \\
\hline
\end{tabular}

TABLE S-V

STATISTICAL RESULTS OF HV AND IGD OBTAINED By CMME AND ITS VARIATIONS, THE BEST VALUES OF EACH ROW ARE HIGHLIGHTED.

\begin{tabular}{|c|c|c|c|c|c|c|c|c|c|c|c|}
\hline \multirow{2}{*}{ Problem } & \multirow{2}{*}{$m$} & & \\
\hline & & $\mathrm{ME}-\mathrm{T}-\mathrm{s}$ & $\mathrm{NEF}=2$ & & & & & & & & \\
\hline \multirow{5}{*}{ C1-DTLZ1 } & & $2617 \mathrm{e}-1(9$ & $610 \mathrm{e}-1(6.89 \mathrm{e}-1$ & $99 \mathrm{e}-1(5)$ & $560 \mathrm{e}-1(1.10 \mathrm{e}-2)=$ & $008 \mathrm{e}-1(8.39 \mathrm{e}-3)$ & $3131 \mathrm{e}-2(4.44 \mathrm{e}-4)$ & $.0949 \mathrm{e}-1(3.45 \mathrm{e}-2)$ & $567 \mathrm{e}-1(2.62 \mathrm{e}-2)$ & $05 \mathrm{e}-2$ & $e-2(2->$ \\
\hline & & e-1 (1. & $9 \mathrm{e}-1(6.60 \mathrm{e}-$ & $59 \mathrm{e}-1(6.07 \mathrm{e}-$ & $36 \mathrm{e}-1(1.24 \mathrm{e}-2)=$ & $5416 \mathrm{e}-1(2.33 \mathrm{e}-2)$ & $142 \mathrm{e}-2(1.45 \mathrm{e}-3$ & $473 \mathrm{e}-1(2.25 \mathrm{e}-2)$ & $579 \mathrm{e}-1(2$. & $69 \mathrm{e}-2(7.5)$ & \\
\hline & & $1(1$. & e- $1(5.52 \mathrm{e}$ & $267 \mathrm{e}-1(5.30$ & $7368 \mathrm{e}-1(2.57 \mathrm{e}-2)=$ & $9.7484 \mathrm{e}-1(2.07 \mathrm{e}-2)$ & $1792 \mathrm{e}-1(3.08$ & $1.3282 \mathrm{e}-1(1.54 \mathrm{e}$ & $1.3871 \mathrm{e}-1(\mathrm{l}$. & $180 \mathrm{e}-1(4$ & $76 e-1$ \\
\hline & & $1(7$. & & & 397e-1 1 & $874 \mathrm{e}-1(1.9$ & & & & & -3) \\
\hline & & $9.8300 \mathrm{e}-1(1.34 \mathrm{e}-2)=$ & $5614 \mathrm{e}-1(5.01 \mathrm{e}-2)$ & $7682 \mathrm{e}-1(4.66 \mathrm{e}-2)$ & $9.5844 \mathrm{e}-1 \quad(2.41 \mathrm{e}-2)$ & $6631 \mathrm{e}-1(3.73 \mathrm{e}-2)$ & $1.4773 \mathrm{e}-1(4.02 \mathrm{e}-3)$ & $2.0632 \mathrm{e}-1(1.58 \mathrm{e}-2)$ & $2.0464 \mathrm{e}-1(1.34 \mathrm{e}-2)$ & $1.2956 \mathrm{e}-1(7.34 \mathrm{e}-3)=$ & $1.2950 \mathrm{e}-1(6.60 \mathrm{e}-3)$ \\
\hline \multirow{4}{*}{ Cl-DTLZ3 } & 3 & $1.5812 \mathrm{e}-1(1.94 \mathrm{e}-1)=$ & & $3.0528 \mathrm{e}-2(9.61 \mathrm{e}-2)$ & $3.8428 \mathrm{e}-2(1.28 \mathrm{e}-1)-$ & $2.4142 \mathrm{e}-1(2.38 \mathrm{e}-1)$ & $3.6890 \mathrm{e}+0(3.88 \mathrm{e}+0)=$ & $6.0366 \mathrm{e}+0(3.38 \mathrm{e}+0)-$ & $7.0060 \mathrm{e}+0(2.6$ & $7.2289 \mathrm{e}+0(2.42 \mathrm{e}+0)-$ & $2.8412 e+0)(3$ \\
\hline & & 2.19 & 0.00 & 0.00 & & 0.0 & 1.1 & 2) - & $1.1070 \mathrm{e}+1$ & $4 \mathrm{e}-2)=$ & $1308 \mathrm{et}$ \\
\hline & & 0.0000 & 0.000 & $\begin{array}{c}+0 \\
0\end{array}$ & 0.00 & 0.0 & 1.1 & 1) - & 1.1 & 1.1 & +0) \\
\hline & & $0.0000 \mathrm{e}+0(0.0$ & 0.000 & ee+0 $(0.00 e+0)=$ & 0.000 & $++0(0$ & 1.3 & 1) - & $\begin{array}{ll}e+1 \\
e+2\end{array}$ & $-1(2.22 \mathrm{e}-2)=$ & $e-2)$ \\
\hline \multirow{5}{*}{ C2-DTLZ2 } & & 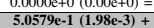 & \begin{tabular}{|l}
$0.0000 \mathrm{e}+0.0(0.00 \mathrm{e}+0)=$ \\
$5.0473 \mathrm{e}-1(2.48 \mathrm{e}-3)+$
\end{tabular} & $\frac{0.0000 \mathrm{e}+0(0.000 \mathrm{e}+0)=}{5.0486 \mathrm{e}-1(2.91 \mathrm{e}-3)+}$ & \begin{tabular}{|l|}
$0.0000 \mathrm{e}+0.0(0)$ \\
$5.0347 \mathrm{e}-1(1)$
\end{tabular} & $\frac{0.0000 \mathrm{e}+0(0.000+0)}{5.0365 \mathrm{e}-1(2.19 \mathrm{e}-3)}$ & $\frac{1.4485 \mathrm{e}+1(3.77 \mathrm{e}-2)-}{5.6203 \mathrm{e}-2(1.40 \mathrm{e}-3)=}$ & $\frac{2.3099 \mathrm{e}+1(3.07 \mathrm{e}+0)-}{5.599 \mathrm{e}-2(1.92 \mathrm{e}-3)}=$ & $\frac{2.2273 \mathrm{e}+1(3.86 \mathrm{e}+0)-}{\mathbf{5} 5794 \mathrm{e}-2(1.57 \mathrm{e}-3)=}$ & $\begin{array}{l}+1(2.84-2)= \\
-2(9.04 \mathrm{e}-4)=\end{array}$ & $\frac{3 e+1(t)}{18 \mathrm{e}-2 \mathrm{c}}$ \\
\hline & & & & $7.1678 \mathrm{e}-1(1$. & & $7.3093 \mathrm{e}-1(1.33 \mathrm{e}-2)$ & $1.7282 \mathrm{e}-1$ & $1.9062 \mathrm{e}-1(1$. & $1.7399 \mathrm{e}-1$ & 1.6 & s. \\
\hline & 8 & $e-3)=$ & & e- $1(1.8$ & $7.8136 \mathrm{e}-1(\mathrm{c}$ & 8.0008e-1 & $2.3640 \mathrm{e}-1$ & $e-1)=$ & 3. & 3) - & 3) \\
\hline & & 8.4 & & $8.5955 \mathrm{e}-1 \quad(8.4$ & -2) $=$ & $8.8249 \mathrm{e}-1$ & 3.1 & $-1)=$ & $3.4065 \mathrm{e}-1$ & $\mathrm{e}-2)=$ & -2) \\
\hline & 15 & $9.5098 \mathrm{e}-1$ & $9.5205 \mathrm{e}-1$ & $9.5289 \mathrm{e}-1(2.37 \mathrm{e}-3)=$ & $9.5276 \mathrm{e}-1$ (1 & $9.5365 \mathrm{e}-1(2$. & $2.3381 \mathrm{e}-1(2.40 \mathrm{e}-2)+$ & e-2) + & $2.3224 \mathrm{e}-1(1$ & $3 e-3)+$ & $\mathrm{e}-2)$ \\
\hline \multirow{4}{*}{ C3-DTLZ4 } & 3 & & & $6.8887 \mathrm{e}-1(1$ & & & & & & & $\frac{e-1)}{1)}(-3(-3$ \\
\hline & & & 9.48 & 9.48 & & & & & & & \\
\hline & & & & $9.9324 \mathrm{e}-1($ & & & & & & & \\
\hline & $\begin{array}{l}10 \\
15\end{array}$ & \begin{tabular}{|l|l}
$9.9896 \mathrm{e}-1(4$ \\
$9.9998-1$ \\
\end{tabular} & $\begin{array}{l}9.98886-1(1 \\
9.9996 \mathrm{e}-1\end{array}$ & $\begin{array}{l}9.98933 \mathrm{e}-1(1.58 \mathrm{e}-4)-)^{-} \\
9.9996 \mathrm{e} 1 \\
(9.33 \mathrm{e}-6)\end{array}$ & \begin{tabular}{|l|l|}
$9.9932 \mathrm{e}-1$ & 3 \\
9 & $99998 \mathrm{e}-1$ \\
11
\end{tabular} & 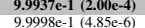 & $\begin{array}{l}6.33 \\
7.33 \\
-13\end{array}$ & $\begin{array}{l}e-3)- \\
e-3)=\end{array}$ & $\begin{array}{l}6.1823 \mathrm{e}-1 \\
7.5506 \mathrm{e}-1 \\
(8)\end{array}$ & $\begin{array}{l}6.0583 \mathrm{e}-1 \\
7.233 \mathrm{e}-1\end{array}$ & (2e-2) \\
\hline \multirow{5}{*}{ DC1-DTLZ1 } & & $6.2399 \mathrm{e}-1$ & $1.8791 \mathrm{e}-1(2$. & $2.5931 \mathrm{e}-1(1$. & \begin{tabular}{|l|l|l}
6.1336 \\
\end{tabular} & 6.169 & & & $2.9245 \mathrm{e}-1$ & $(\mathrm{e}-4)=$ & \\
\hline & & 7.61 & & 1 & & & 5.0 & & 3.3 & & \\
\hline & & 7.90 & 2.07 & te-1 $(1$. & 7.8 & 7.9 & 8.2 & 3.4 & 3. & 8.0 & 3) \\
\hline & & & & 1.8 & & & & & 3) - & & -3) \\
\hline & 15 & 7.95 & 1.89 & 2.19 & & 7.89 & & & & & \\
\hline \multirow{5}{*}{ DCI-DTLZ3 } & 3 & 4.294 & & 3.03 & & & & & & & \\
\hline & & 2. & $=$ & 4.76 & 4.46 & & & & & & \\
\hline & 8 & & & & & & & & & & \\
\hline & & & & 1. & & & & & & & \\
\hline & $\frac{15}{3}$ & $\frac{4.24}{8.22}-x-1$ & & 4.4. & \begin{tabular}{|l|l|}
$\mathbf{5 . 7 4}$ \\
7.78 \\
\end{tabular} & & & & $-\frac{-1)-}{-2)-}$ & $\begin{array}{l}-2)= \\
-2)= \\
\end{array}$ & $\frac{e-1)}{-2)}$ \\
\hline \multirow{4}{*}{ DC2-DTLZ1 } & & & & 8. & 8. & & & 1. & 1. & & -2) \\
\hline & & & & 0 & & & & & & & \\
\hline & & & & & & & & & & & \\
\hline & & & & & & & & & & & \\
\hline \multirow{5}{*}{ DC2-DTLZ3 } & 3 & & & & & & & & & & \\
\hline & & & & & & & & & & & \\
\hline & 8 & 2.5 & & 1. & & & & & & & \\
\hline & 10 & & & 3. & & & & & & & \\
\hline & & $\frac{1.11}{2.19}$ & $\frac{-2)=}{-1)-}$ & $\frac{1.2532 \mathrm{e}-2 \mathrm{t}}{1.688 \mathrm{e}-1 \mathrm{~s}}$ & \begin{tabular}{|l|}
3.80 \\
2.42 \\
\end{tabular} & & $\frac{9.2}{1.6}$ & & 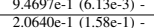 & & \\
\hline \multirow{4}{*}{ DC3-DTLZ1 } & & 3.485 & & 1.4971 & 2.2344 & $2.4335 \mathrm{e}-2$ & 1.19 & 3.6 & -1) - & -1) - & $2 e-1)$ \\
\hline & & & & $\mathrm{Na}$ & & $\mathrm{N}$ & $\mathrm{N}$ & $\mathrm{Na}$ & & $\mathrm{N}$ & \\
\hline & & & & & & & & & & & \\
\hline & & & & & & & & & & & \\
\hline \multirow{4}{*}{ DC3-DTLZ3 } & & $=$ & & & & & & & & & \\
\hline & & & & & & & & & & & \\
\hline & 8 & & & 0.0 & & & & & & & \\
\hline & & $\begin{array}{l}0.00000+0+0(0.00 \mathrm{e}+0)= \\
0.0000 \mathrm{e}+0.000 \mathrm{e}+0)\end{array}$ & $\begin{array}{l}0.00000+0+0(0.00 \mathrm{e}+0)= \\
0.000 \mathrm{e}+0(0.00 \mathrm{e}+0)\end{array}$ & $\begin{array}{l}0.0000 \mathrm{e}+0+(0.00 \mathrm{e} e+0)= \\
0.000 \mathrm{e}+0(0.00 \mathrm{e}+0)=\end{array}$ & $\mid \begin{array}{l}0.0000 \mathrm{e}+0(0.00 \mathrm{e}+0)= \\
0.0000 \mathrm{e}+0.00 \mathrm{e}+0)=\end{array}$ & $0.0000 \mathrm{e}+0(0.00 \mathrm{e}+0$ & $\begin{array}{l}7.5602 \mathrm{e} e+(2.32 \mathrm{e} e+0)- \\
3.9929+0+0(1.18 \mathrm{e}+0)\end{array}$ & $\begin{array}{l}5.08030+0(1.87+e+0)- \\
6.6416 \mathrm{e}+0+(2.23+0)-\end{array}$ & $\begin{array}{l}4.7 .433+0+(1.444 \mathrm{e}+0)- \\
6.4936 \mathrm{e}+0(2.00 \mathrm{e}+0)\end{array}$ & $\begin{array}{l}1.75832 \mathrm{e}+0(3.77 \mathrm{e}-1)= \\
2.0362 \mathrm{e}+0.5 .28 \mathrm{e}-1)\end{array}$ & $\begin{array}{l}1.7966 \mathrm{e}+0(8.425-1) \\
1.5419 \mathrm{e}+0 \\
(7.19 \mathrm{e}-1)\end{array}$ \\
\hline & $9 / 17 / 24$ & $2 / 30$ & $2 / 30 / 18$ & $4 / 7 /:$ & & $9 / 24$ & $3 / 3 \S$ & $4 / 3$ & $3 / 9$ & \\
\hline
\end{tabular}




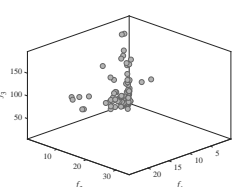

(a)

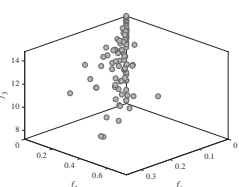

(b)

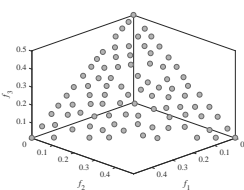

(c)

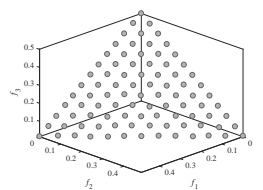

(d)

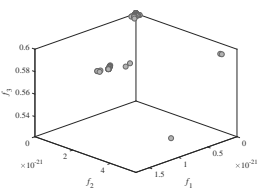

(e)

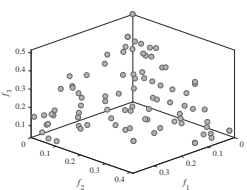

(f)

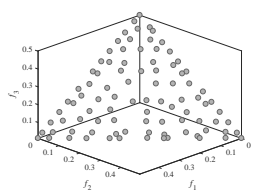

(g)

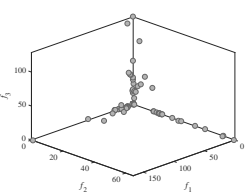

(h)

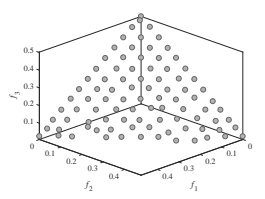

(i)

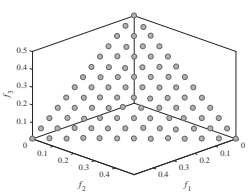

(j)

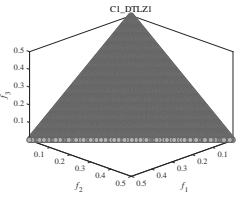

(k)

Fig. S-1. Feasible and nondominated solutions with the median HV value among 30 runs on 3-objective C1-DTLZ1. (a) NSGA-II-ToR, (b) ToP, (c) CCMO, (d) NSGA-III, (e) I-DBEA, (f) PPS, (g) C-TAEA, (h) TiGE-2, (i) DCNSGA-III, (j) CMME, and (k) true PF.

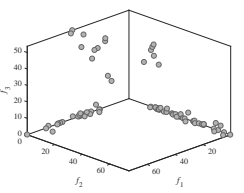

(a)

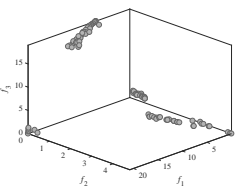

(b)

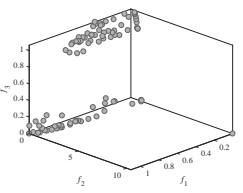

(c)

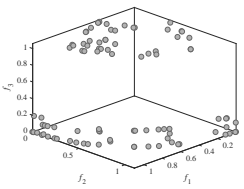

(d)

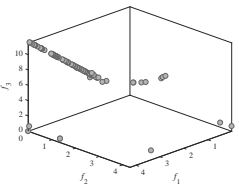

(e)

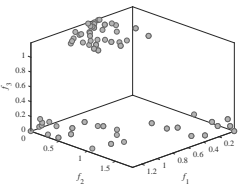

(f)

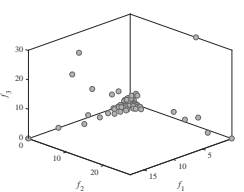

(g)

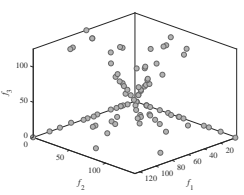

(h)

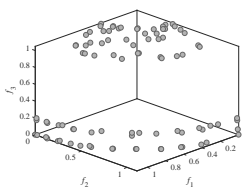

(i)

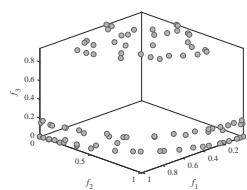

(j)

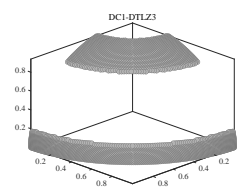

(k)

Fig. S-2. Feasible and nondominated solutions with the median HV value among 30 runs on 3-objective DC1-DTLZ3. (a) NSGA-II-ToR, (b) ToP, (c) CCMO, (d) NSGA-III, (e) I-DBEA, (f) PPS, (g) C-TAEA, (h) TiGE-2, (i) DCNSGA-III, (j) CMME, and (k) true PF.

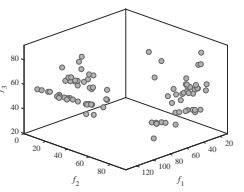

(a)

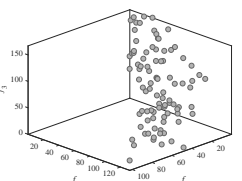

(b)

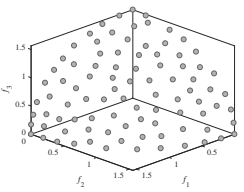

(c)

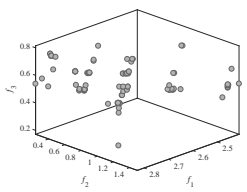

(d)

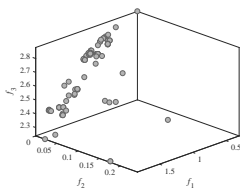

(e)

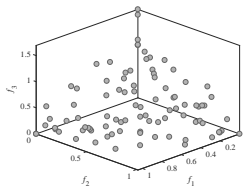

(f)

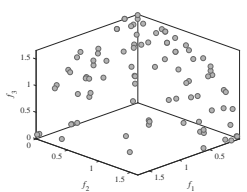

(g)

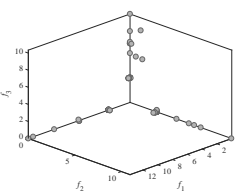

(h)

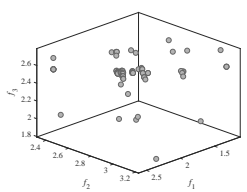

(i)

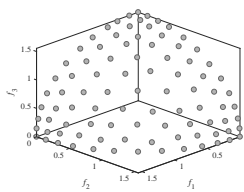

(j)

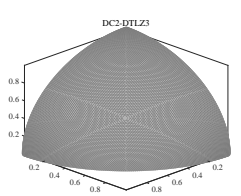

(k)

Fig. S-3. Feasible and nondominated solutions with the median HV value among 30 runs on 3-objective DC2-DTLZ3. (a) NSGA-II-ToR, (b) ToP, (c) CCMO, (d) NSGA-III, (e) I-DBEA, (f) PPS, (g) C-TAEA, (h) TiGE-2, (i) DCNSGA-III, (j) CMME, and (k) true PF. 


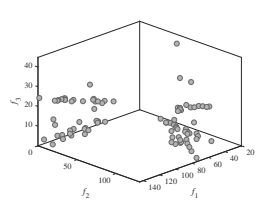

(a)

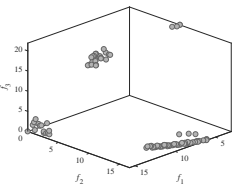

(b)

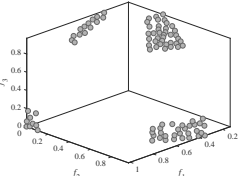

(c)

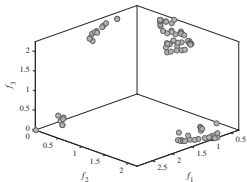

(d)

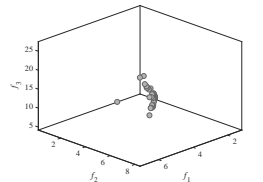

(e)

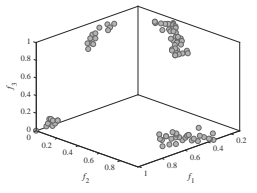

(f)

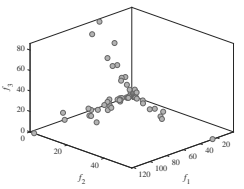

(g)

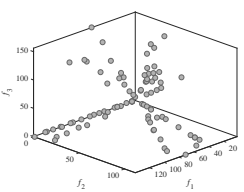

(h)

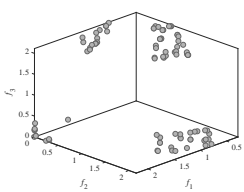

(i)

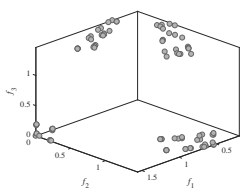

(j)

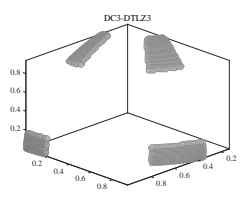

(k)

Fig. S-4. Feasible and nondominated solutions with the median HV value among 30 runs on 3-objective DC3-DTLZ3. (a) NSGA-II-ToR, (b) ToP, (c) CCMO, (d) NSGA-III, (e) I-DBEA, (f) PPS, (g) C-TAEA, (h) TiGE-2, (i) DCNSGA-III, (j) CMME, and (k) true PF.

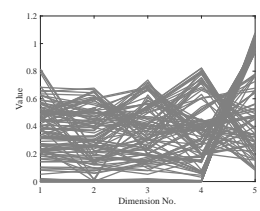

(a)

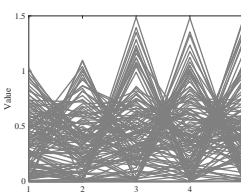

(b)

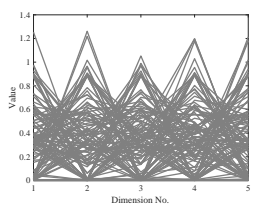

(c)

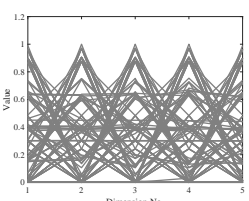

(d)

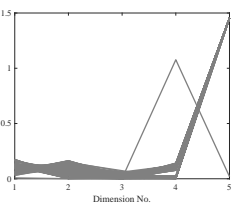

(e)

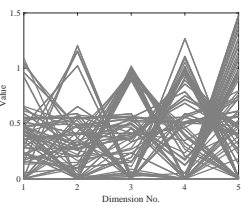

(f)

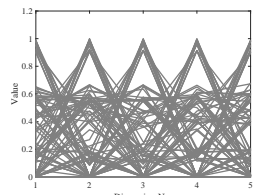

(g)

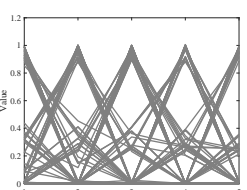

(h)

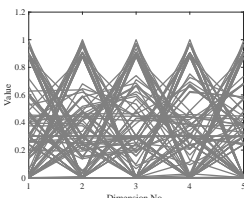

(i)

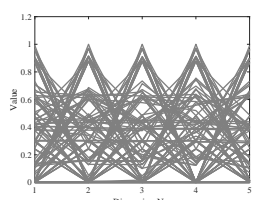

(j)

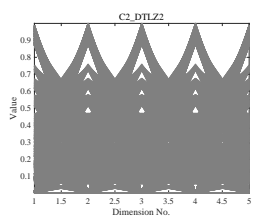

$(\mathrm{k})$

Fig. S-5. Feasible and nondominated solutions with the median HV value among 30 runs on 5-objective C2-DTLZ2. (a) NSGA-II-ToR, (b) ToP, (c) CCMO, (d) NSGA-III, (e) I-DBEA, (f) PPS, (g) C-TAEA, (h) TiGE-2, (i) DCNSGA-III, (j) CMME, and (k) true PF.

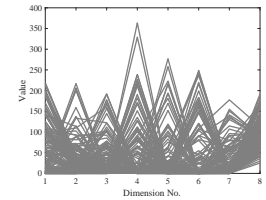

(a)

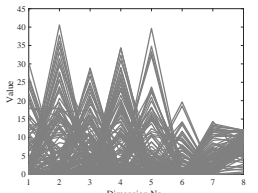

(b)

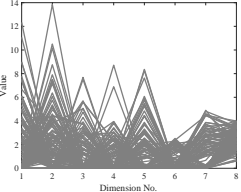

(c)

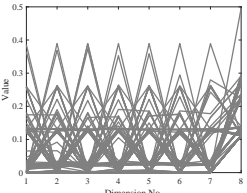

(d)

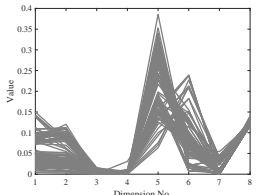

(e)

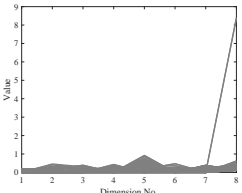

(f)

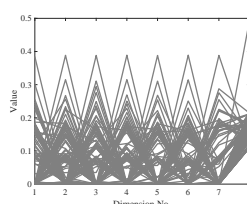

(g)

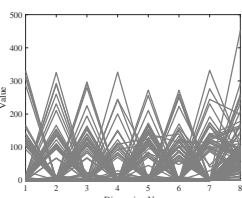

(h)

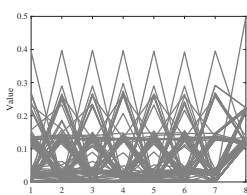

(i)

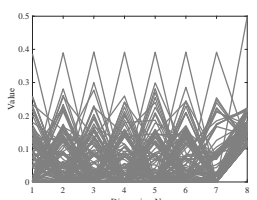

(j)

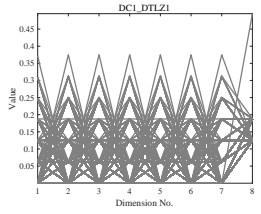

(k)

Fig. S-6. Feasible and nondominated solutions with the median HV value among 30 runs on 8-objective DC1-DTLZ1. (a) NSGA-II-ToR, (b) ToP, (c) CCMO, (d) NSGA-III, (e) I-DBEA, (f) PPS, (g) C-TAEA, (h) TiGE-2, (i) DCNSGA-III, (j) CMME, and (k) true PF. 


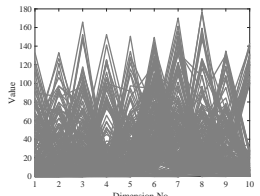

(a)

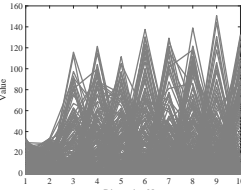

(b)

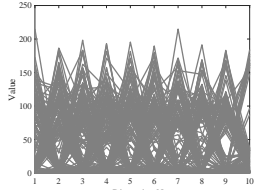

(c)

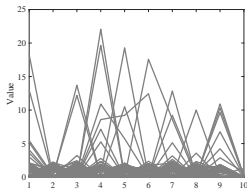

(d)

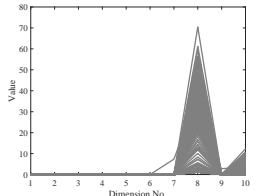

(e)

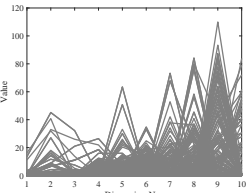

(f)

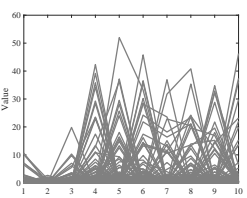

(g)

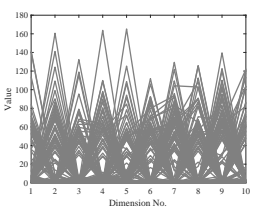

(h)

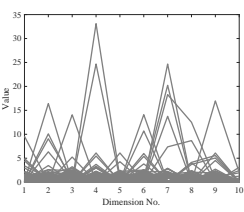

(i)

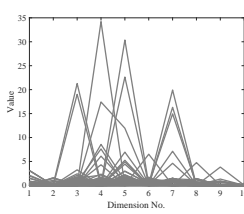

(j)

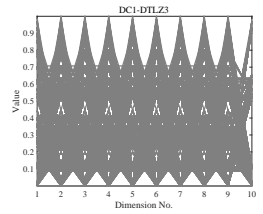

(k)

Fig. S-7. Feasible and nondominated solutions with the median HV value among 30 runs on 10-objective DC1-DTLZ3. (a) NSGA-II-ToR, (b) ToP, (c) CCMO, (d) NSGA-III, (e) I-DBEA, (f) PPS, (g) C-TAEA, (h) TiGE-2, (i) DCNSGA-III, (j) CMME, and (k) true PF.

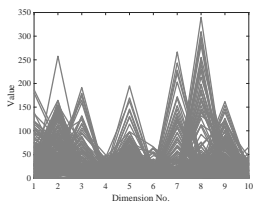

(a)

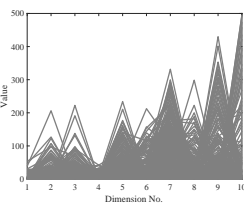

(b)

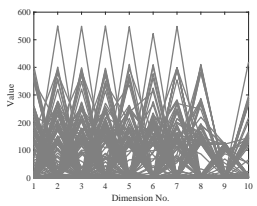

(c)

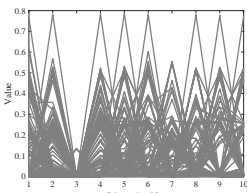

(d)

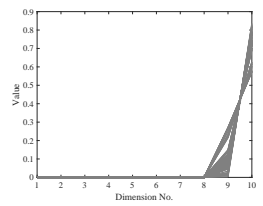

(e)

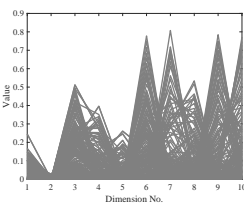

(f)

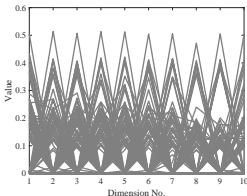

(g)

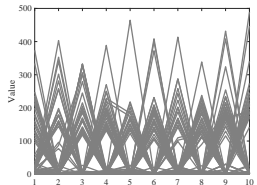

(h)

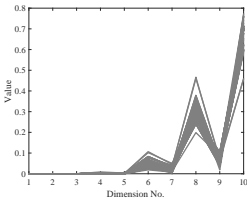

(i)

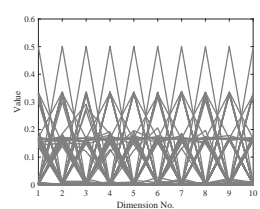

(j)

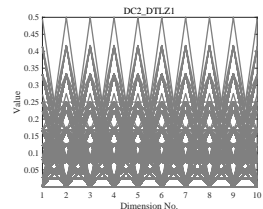

(k)

Fig. S-8. Feasible and nondominated solutions with the median HV value among 30 runs on 10-objective DC2-DTLZ1. (a) NSGA-II-ToR, (b) ToP, (c) CCMO, (d) NSGA-III, (e) I-DBEA, (f) PPS, (g) C-TAEA, (h) TiGE-2, (i) DCNSGA-III, (j) CMME, and (k) true PF.

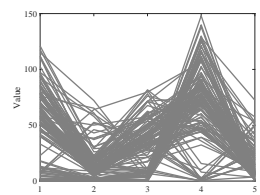

(a)

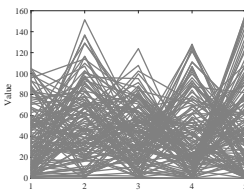

(b)

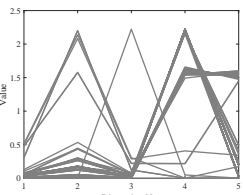

(c)

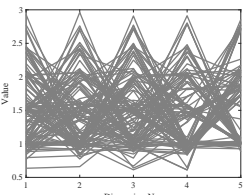

(d)

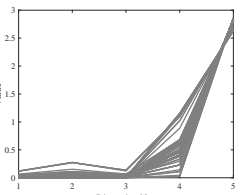

(e)

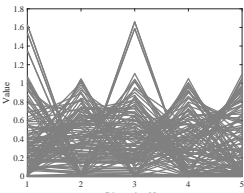

(f)

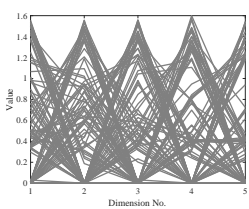

(g)

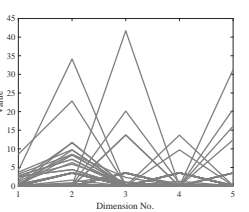

(h)

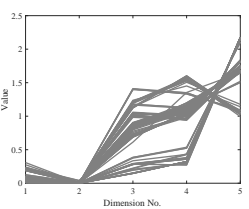

(i)

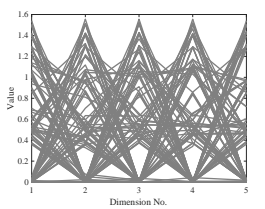

(j)

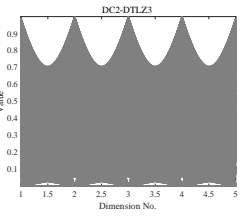

(k)

Fig. S-9. Feasible and nondominated solutions with the median HV value among 30 runs on 5-objective DC2-DTLZ3. (a) NSGA-II-ToR, (b) ToP, (c) CCMO, (d) NSGA-III, (e) I-DBEA, (f) PPS, (g) C-TAEA, (h) TiGE-2, (i) DCNSGA-III, (j) CMME, and (k) true PF. 


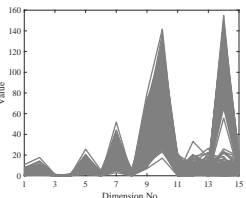

(a)

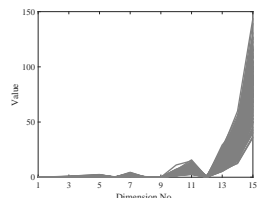

(b)

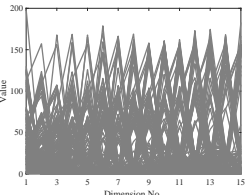

(c)

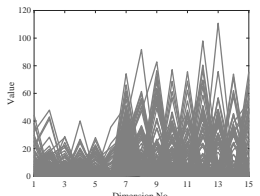

(d)

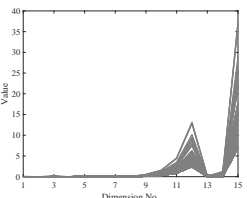

(e)

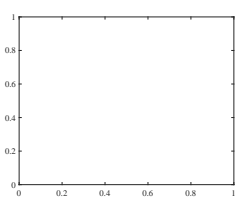

(f)

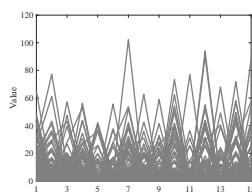

(g)

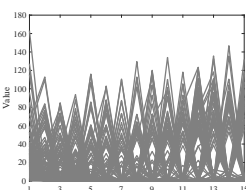

(h)

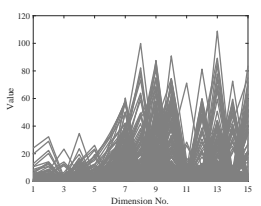

(i)

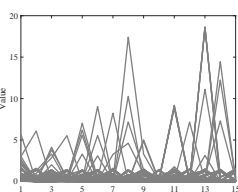

(j)

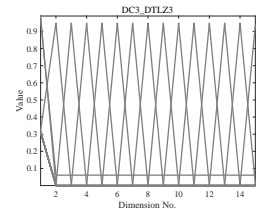

(k)

Fig. S-10. Feasible and nondominated solutions with the median HV value among 30 runs on 15-objective DC3-DTLZ3. (a) NSGA-II-ToR, (b) ToP, (c) CCMO, (d) NSGA-III, (e) I-DBEA, (f) PPS, (g) C-TAEA, (h) TiGE-2, (i) DCNSGA-III, (j) CMME, and (k) true PF.

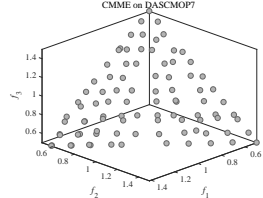

(a)

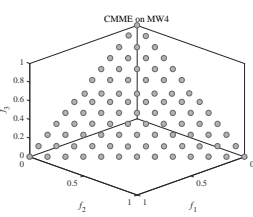

(g)

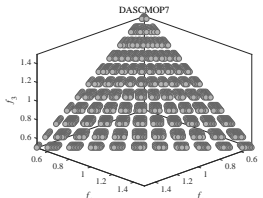

(b)

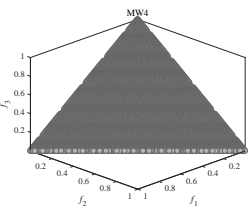

(h)

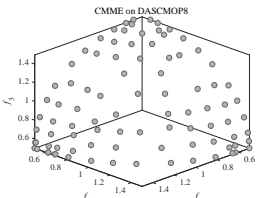

(c)

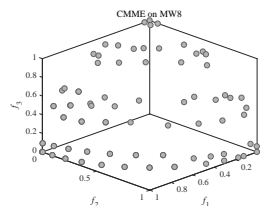

(i)

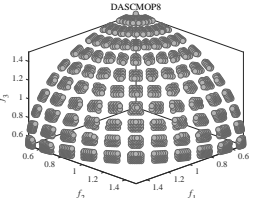

(d)

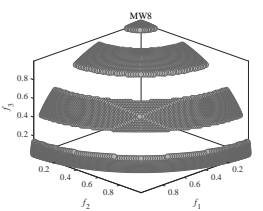

(j)

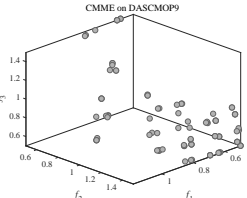

(e)

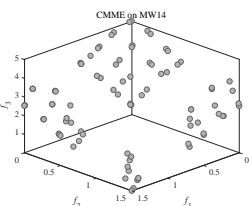

(k)

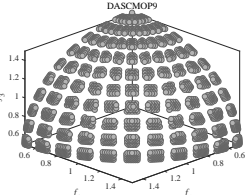

(f)

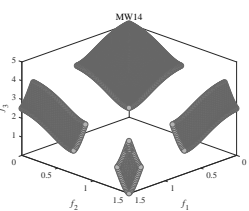

(1)

Fig. S-11. Feasible and nondominated solutions with the median HV value among 30 runs on 3-objective DAS-CMOP and MW instances obtained by CMME.

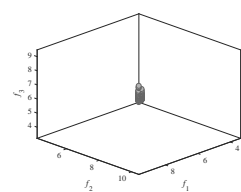

(a)

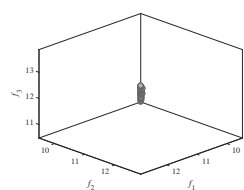

(b)

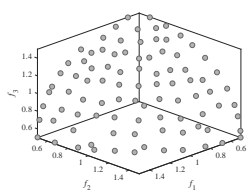

(c)

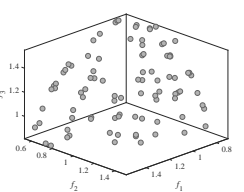

(d)

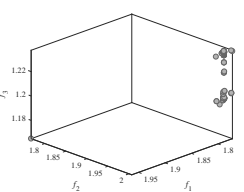

(e)

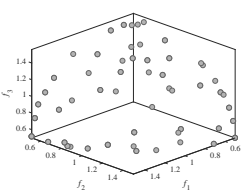

(f)

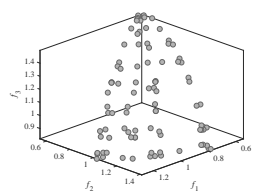

(g)

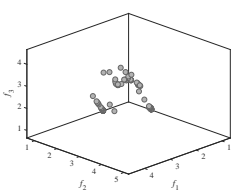

(h)

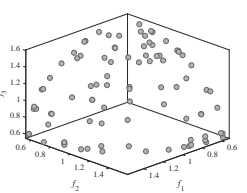

(i)

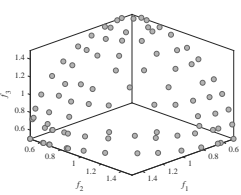

(j)

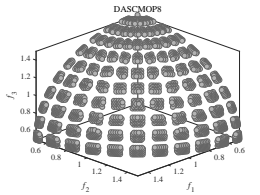

(k)

Fig. S-12. Feasible and nondominated solutions with the median HV value among 30 runs on 3-objective DAS-CMOP8. (a) NSGA-II-ToR, (b) ToP, (c) CCMO, (d) NSGA-III, (e) I-DBEA, (f) PPS, (g) C-TAEA, (h) TiGE-2, (i) DCNSGA-III, (j) CMME, and (k) true PF. 


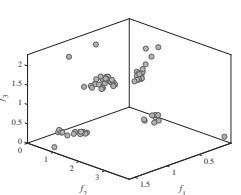

(a)

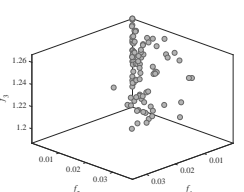

(b)

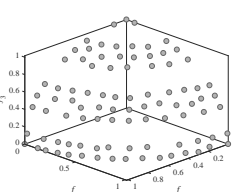

(c)

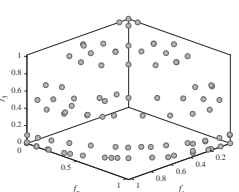

(d)

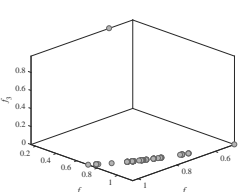

(e)

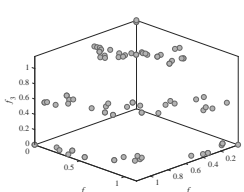

(f)

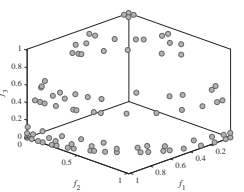

(g)

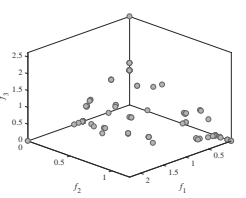

(h)

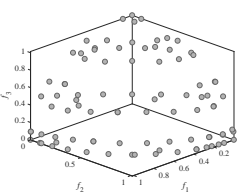

(i)

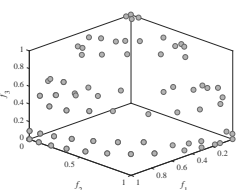

(j)

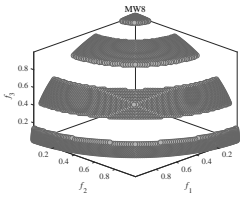

(k)

Fig. S-13. Feasible and nondominated solutions with the median HV value among 30 runs on 3-objective MW8. (a) NSGA-II-ToR, (b) ToP, (c) CCMO, (d) NSGA-III, (e) I-DBEA, (f) PPS, (g) C-TAEA, (h) TiGE-2, (i) DCNSGA-III, (j) CMME, and (k) true PF.

TABLE S-VI

HV OF CMME AND CMOEAS/CMAOEAS IN COMPARISON ON RW-MOP.

\begin{tabular}{|c|c|c|c|c|c|c|c|c|c|c|}
\hline Problem & NSGA-II-ToR & ToP & СCMO & NSGA-III & I-DBEA & PPS & C-TAEA & TiGE-2 & DCNSGA-III & CMME \\
\hline Gear Box Design & $8.7162 \mathrm{e}-2(2.80 \mathrm{e}-3)-$ & $8.9136 \mathrm{e}-2(2.03 \mathrm{e}-4)-$ & $8.8747 \mathrm{e}-2(2.29 \mathrm{e}-4)-$ & $9.0018 \mathrm{e}-2(2.67 \mathrm{e}-4)+$ & $1.8666 \mathrm{e}-2(1.89 \mathrm{e}-2)-$ & $8.9439 \mathrm{e}-2(2.81 \mathrm{e}-4) \approx$ & $8.7481 \mathrm{e}-2(3.22 \mathrm{e}-3)-$ & $8.6687 \mathrm{e}-2(8.52 \mathrm{e}-4)-$ & $7.9700 \mathrm{e}-2(1.26 \mathrm{e}-2)-$ & $8.9287 \mathrm{e}-2(1.19 \mathrm{e}-3)$ \\
\hline $\begin{array}{l}\text { Multi-product Batch Plant } \\
\end{array}$ & $2.2464 \mathrm{e}-1(1.87 \mathrm{e}-2)-$ & $3.0167 \mathrm{e}-1(6.31 \mathrm{e}-2) \approx$ & $3.1838 \mathrm{e}-1(1.09 \mathrm{e}-2)-$ & $3.2279 \mathrm{e}-1(1.39 \mathrm{e}-2)-$ & $1.6397 \mathrm{e}-1(6.54 \mathrm{e}-2)-$ & $3.0697 \mathrm{e}-1(3.05 \mathrm{e}-2)-$ & $1.3558 \mathrm{e}-1(6.86 \mathrm{e}-2)-$ & $3.1729 \mathrm{e}-1(1.66 \mathrm{e}-2)-$ & $3.0150 \mathrm{e}-1(4.03 \mathrm{e}-2)-$ & $3.2988 \mathrm{e}-1(1.27 \mathrm{e}-2)$ \\
\hline Heat Exchanger Network Design & $\mathrm{NaN}(\mathrm{NaN})$ & $\mathrm{NaN}(\mathrm{NaN})$ & $\mathrm{NaN}(\mathrm{NaN})$ & $\mathrm{NaN}(\mathrm{NaN})$ & $\mathrm{NaN}(\mathrm{NaN})$ & $7.7253 \mathrm{e}-1(2.98 \mathrm{e}-1)-$ & NaN (NaN) & $1.0034 \mathrm{e}+0(4.74 \mathrm{e}-3) \approx$ & $\mathrm{NaN}(\mathrm{NaN})$ & $1.0083 \mathrm{e}+0(1.43 \mathrm{e}-2)$ \\
\hline$+1-1 \approx$ & $0 / 3 / 0$ & $0 / 2 / 1$ & $0 / 3 / 0$ & $1 / 2 / 0$ & $0 / 3 / 0$ & $0 / 2 / 1$ & $0 / 3 / 0$ & $0 / 2 / 1$ & $0 / 3 / 0$ & \\
\hline
\end{tabular}

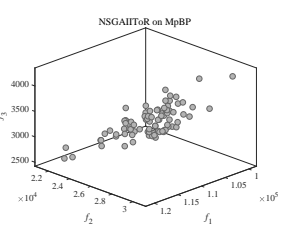

(a)

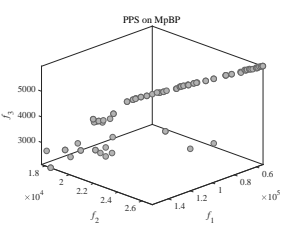

(f)

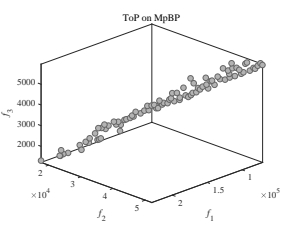

(b)

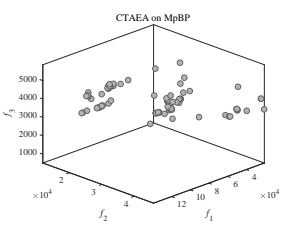

(g)

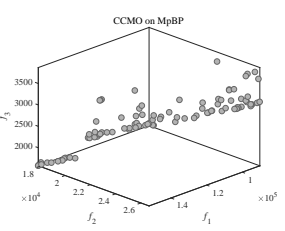

(c)

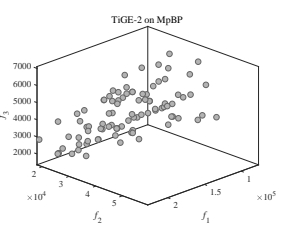

(h)

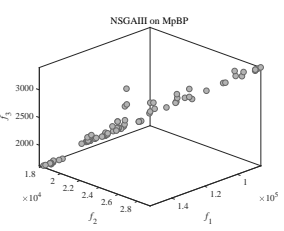

(d)

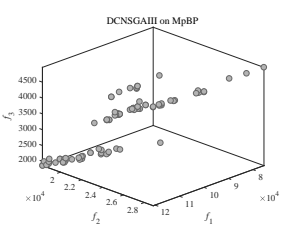

(i)

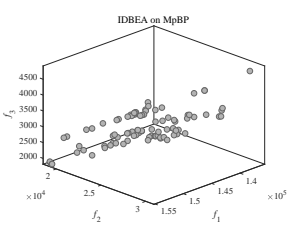

(e)

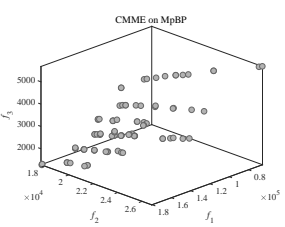

(j)

Fig. S-14. Feasible and nondominated solutions with the median HV value among 30 runs on Multi-product Batch Plant problem. (a) NSGA-II-ToR, (b) ToP, (c) CCMO, (d) NSGA-III, (e) I-DBEA, (f) PPS, (g) C-TAEA, (h) TiGE-2, (i) DCNSGA-III and (j) CMME. 